\title{
Theta Oscillations in the Medial Prefrontal Cortex Are Modulated by Spatial Working Memory and Synchronize with the Hippocampus through Its Ventral Subregion
}

\author{
Pia-Kelsey O'Neill, ${ }^{1,2}$ Joshua A. Gordon, ${ }^{1,2,3}$ and Torfi Sigurdsson ${ }^{1,4}$ \\ ${ }^{1}$ Department of Psychiatry and ${ }^{2}$ Graduate Program in Neurobiology and Behavior, Columbia University, New York, New York 10032, ${ }^{3}$ Division of \\ Integrative Neuroscience, New York State Psychiatric Institute, New York, New York 10032, and ${ }^{4}$ Institute of Neurophysiology, Neuroscience Center, \\ Goethe University, 60590 Frankfurt, Germany
}

\begin{abstract}
The rodent medial prefrontal cortex (mPFC) is critical for spatial working memory (SWM), but the underlying neural processes are incompletely understood. During SWM tasks, neural activity in the mPFC becomes synchronized with theta oscillations in the hippocampus, and the strength of hippocampal-prefrontal synchrony is correlated with behavioral performance. However, to what extent the mPFC generates theta oscillations and whether they are also modulated by SWM remains unclear. Furthermore, it is not known how theta oscillations in the mPFC are synchronized with theta oscillations in the hippocampus. Although the ventral hippocampus (vHPC) projects directly to the MPFC, previous studies have only examined synchrony between the mPFC and the dorsal hippocampus (dHPC), with which it is not directly connected. To address these issues, we recorded simultaneously from the dHPC, vHPC, and mPFC of mice performing a SWM task in a T-maze. The local field potential recorded in the MPFC displayed robust theta oscillations that were reflected in local measures of neuronal activity and modulated by SWM performance. mPFC theta oscillations were also synchronized with theta oscillations in both the vHPC and dHPC, and the magnitude of theta synchrony was modulated by SWM. Removing the influence of the vHPC either computationally (through partial correlations) or experimentally (through pharmacological inactivation) reduced theta synchrony between the MPFC and dHPC. These results reveal theta oscillations as a prominent feature of neural activity in the mPFC and a candidate neural mechanism underlying SWM. Furthermore, our results suggest that the vHPC plays a major role in synchronizing theta oscillations in the MPFC and the hippocampus.
\end{abstract}

\section{Introduction}

Spatial working memory (SWM), the ability to remember spatial locations in the short term, is a critical cognitive function. For example, efficient foraging requires animals to keep track of which areas of their environment have already been visited and which ones have not (Haupt et al., 2010). In the laboratory, SWM can be assessed using various maze-based tasks, such as the radial arm maze and the T-maze (Aultmann and Moghaddam, 2001). The rodent medial prefrontal cortex ( $\mathrm{mPFC}$ ) has emerged as a critical structure for successful performance on these tasks. Le-

\footnotetext{
Received June 5, 2013; revised July 19, 2013; accepted July 26, 2013.

Author contributions: P.-K.O., J.A.G., and T.S. designed research;P.-K.O. and T.S. performed research;P.-K.O. and T.S. analyzed data; P.-K.O., J.A.G., and T.S. wrote the paper.

T.S. was supported by a Sackler Foundation Fellowship in Developmental Psychobiology, a National Alliance for Research on Schizophrenia and Depression Young Investigator Award, and a Neuronal Coordination Research Focus Frankfurt grant through the Initiative for Scientific and Economic Excellence of the Land Hessen (LOEWE).P.-K.O. was supported by National Institutes of Health (NIH) Training Grant T32 NS064928. J.A.G. was supported by NIH Grant R01 MH081968, a Rising Star Award from the International Mental Health Research Organization, and a Research Grant from the Hope for Depression Research Foundation. We thank A. Adhikari for discovering the correlations between $\mathrm{dHPC} / \mathrm{mPFC}$ and $\mathrm{dHPC} / \mathrm{vHPC}$ coherences and E. D. Leonardo and members of the Gordon laboratory for helpful comments on this manuscript.

The authors declare no competing financial interests.

Correspondence should be addressed to Joshua Gordon, Department of Psychiatry, Columbia University, 1051 Riverside Drive, Unit 87, Kolb Annex, Room 136, New York, NY 10032. E-mail: jg343@columbia.edu.

DOI:10.1523/JNEUROSCI.2378-13.2013

Copyright $\odot 2013$ the authors $\quad 0270-6474 / 13 / 3314211-14 \$ 15.00 / 0$
}

sioning or inactivating the mPFC impairs the acquisition of SWM tasks if done before training as well as task performance if done after training (for review, see Dalley et al., 2004). However, the exact neural processes within the $\mathrm{MPFC}$ that underlie SWM are incompletely understood. Individual mPFC neurons display a diversity of task-related firing patterns during SWM performance (Jung et al., 1998). Furthermore, neural activity in the mPFC becomes synchronized with activity in the hippocampus during SWM tasks, and the strength of this synchrony correlates with animals' behavioral performance (Jones and Wilson 2005; Hyman et al., 2010; Sigurdsson et al., 2010). Lesion studies suggest that such hippocampal-prefrontal interactions are critical for successful task performance (Floresco et al., 1997; Wang and Cai 2006; Izaki et al., 2008).

Oscillatory activity plays a crucial role in enabling inter-areal interactions in the brain (Fries, 2009). In the case of hippocampal-prefrontal interactions, theta oscillations play a particularly important role (Benchenane et al., 2011; Colgin 2011; Gordon 2011). The firing of mPFC neurons is modulated by the phase of theta oscillations in the hippocampus, and the local field potentials (LFPs) in the two structures display coherence in the thetafrequency range (Jones and Wilson 2005; Siapas et al., 2005; Sirota et al., 2008; Benchenane et al., 2010; Hyman et al., 2010; Sigurdsson et al., 2010). However, whether the mPFC itself generates theta oscillations and whether these are modulated by 
SWM has not been rigorously examined. Furthermore, it remains unclear how hippocampal-prefrontal theta synchrony is mediated, because only some of its subdivisions project directly to the mPFC. In particular, the dorsal subdivision of the hippocampus, on which most studies to date have focused, does not project directly to the $\mathrm{mPFC}$, whereas the ventral subdivision does (Dégenètais et al., 2003; Hoover and Vertes 2007).

To address these issues, we recorded neural activity from the mPFC, as well as from the dorsal (dHPC) and ventral (vHPC) subregions of the hippocampus, in mice performing the T-maze delayed non-match-to-sample test of SWM. We found that theta oscillations are a prominent feature of the MPFC LFP and are reflected in local measures of neuronal activity. mPFC theta oscillations also displayed robust modulation by the mnemonic demands of the working memory task and correlated with behavioral performance. Although mPFC theta oscillations were synchronized to both dorsal and ventral hippocampal theta, our analysis suggests that hippocampal-prefrontal synchrony may rely primarily on activity in the vHPC. This was confirmed by inactivating the vHPC, which led to a reduction in hippocampalprefrontal theta coherence. Together, these data demonstrate the existence of local theta-frequency oscillations in the mPFC, which are modulated by SWM and synchronized with the hippocampus via its ventral subregion.

\section{Materials and Methods}

Animals. Twelve male wild-type C57BL/6 mice (3-6 months old) were used in the electrophysiological study, three of which were obtained from The Jackson Laboratory and nine of which were bred at Columbia University. Because of missed placements, only five C57BL/6 mice could be used for the analysis of vHPC activity. Therefore, we added to our dataset of vHPC activity five mice of the $129 \mathrm{~Sv} / \mathrm{Ev}$ strain. No differences were found between strains. For the inactivation experiments, an additional 12 C57BL/6 mice from Jackson were used. The procedures described here were conducted in accordance with National Institutes of Health regulations and approved by the Columbia University and New York State Psychiatric Institute Institutional Animal Care and Use Committees.

Surgical procedures. Animals were initially anesthetized with ketamine/ xylazine, placed in a stereotaxic frame, and maintained on isoflurane for the duration of the surgery. Craniotomies were made to allow two tetrodes or five stereotrodes to be implanted in the $\mathrm{mPFC}(1.7 \mathrm{~mm}$ anterior to bregma, $0.4 \mathrm{~mm}$ lateral to the midline, and $1.5 \mathrm{~mm}$ below the brain surface) and tungsten wires to be placed into the dHPC (1.94 $\mathrm{mm}$ posterior to bregma, $1.5 \mathrm{~mm}$ lateral to the midline, and $1.4 \mathrm{~mm}$ below the brain surface) and vHPC ( $3.16 \mathrm{~mm}$ posterior to bregma, $3.2 \mathrm{~mm}$ lateral to the midline, and $4.1 \mathrm{~mm}$ below the brain surface). Skull screws overlying the cerebellum and frontal cortex served as ground and reference, respectively. All wires were connected to a 16-channel electrode interface board, and the mPFC electrodes were anchored to a microdrive that made it possible to advance them along the dorsoventral axis. To verify recording locations, current $(50 \mathrm{~mA}, 10 \mathrm{~s})$ was passed through the electrodes at the end of the experiment. Animals were then perfused, and the brains were cut on a cryostat and stained for Nissl bodies using cresyl violet.

Behavior. Animals were trained on a discrete non-match-to-sample T-maze test of SWM (see Fig. $3 A$ ) as described previously (Sigurdsson et al., 2010). Each trial of the task consisted of two phases. In the "sample phase," mice ran down the center arm of the maze and were directed into one of the goal arms by a barrier that closed off the other arm. After a brief delay $(\sim 10 \mathrm{~s})$, the mice again ran down the center arm but now had to choose between two open goal arms ("choice phase"). To obtain a reward, animals were required to enter the goal arm not visited during the sample phase. After $2 \mathrm{~d}$ of habituation to the maze and $2 \mathrm{~d}$ of shaping, animals were given daily training sessions of 10 trials until they reached criterion performance, defined as performance of at least seven trials correct per day for 3 consecutive days. After this criterion had been reached, animals were given daily sessions of $20-25$ trials. Although neural data were recorded throughout the experiment, the results presented here are from postcriterion sessions. mPFC electrodes were advanced regularly to ensure that different populations of cells were recorded in each behavioral session.

Data acquisition. Neural data (putative spikes and field potentials) were acquired using a 16-channel head stage and a Cheetah data acquisition system (Neuralynx). The animal's position in the T-maze was monitored using two light-emitting diodes mounted on the head stage. To extract putative spikes, neural signals were bandpass filtered between 0.6 and $6 \mathrm{kHz}$, and waveforms that passed a threshold were digitized at 30 $\mathrm{kHz}$. Waveforms were then sorted offline into single-unit clusters using SPIKESORT3D (Neuralynx). To extract field potential activity, the same signals were bandpass filtered between 1 and $1000 \mathrm{~Hz}$ and digitized at 2 $\mathrm{kHz}$. Additional analysis of neural and behavioral data was performed using custom-written MATLAB scripts (MathWorks).

Field potential analysis. To reveal oscillatory activity in the mPFC, the LFP was convolved with a series of Morlet wavelets with center frequencies ranging from 1 to $100 \mathrm{~Hz}$ and a length of two cycles. The result of these convolutions is the wavelet transform of the $\operatorname{LFP}, \operatorname{WTLFP}(f, t)$, a matrix of complex numbers (or vectors) whose absolute values (or length) and arguments (or angles) represent the amplitude and phase, respectively, of the LFP at frequency $f$ and time $t$. Therefore, the absolute values of WTLFP represent fluctuations in frequency band-limited power (BLP) over time (Fig. 1A). One can then ask whether fluctuations in BLP themselves display any periodicity (Leopold et al., 2003). To examine this, we computed the wavelet transform of BLP at each frequency (i.e., of each row of the spectrogram in Fig. 1A), using the same wavelet parameters as described above. Averaging the absolute values of $\operatorname{WTBLP}\left(f_{\mathrm{BLP}}, f, t\right)$ over time yields the wavelet power spectrum of BLP at each frequency, an example of which is shown in Figure 1C. Each row of this plot represents the power spectrum of fluctuations in BLP at each frequency (showing, for example, that power at $60 \mathrm{~Hz}$ fluctuates with theta periodicity). To further examine whether oscillations in BLP were coherent with the raw LFP, we computed the cross-spectra of $\mathrm{WT}_{\mathrm{LFP}}$ and $\mathrm{WT}_{\mathrm{BLP}}$, averaged them over time, and normalized them to the product of the time-averaged absolute values of $\mathrm{WT}_{\mathrm{LFP}}$ and $\mathrm{WT}_{\mathrm{BLP}}$. This yielded the coherence of the BLP at each frequency to the raw LFP, an example of which is shown in Figure 1E. To summarize the spectral analysis of BLP across animals, we first averaged for each animal the BLP power spectra and coherences between 40 and $60 \mathrm{~Hz}$. The resulting power spectra and coherences were then normalized to their maximum value before being averaged across animals (Fig. $1 D, F$ ).

To measure coordination of theta activity in the MPFC and the hippocampus, we measured both the coherence of the LFPs in the two structures and their power correlations. Coherence was computed using the multitaper method (MATLAB routines provided by K. Harris, University College London, London, UK, and G. Buzsáki, New York University, New York, NY). Field potentials were divided into $500 \mathrm{~ms}$ segments (50 ms overlap), and the Fourier transform of each segment was computed after being multiplied by two orthogonal data tapers. Coherence was then computed by averaging the cross-spectral densities of two field potential signals across data windows and tapers and normalizing to the power spectral densities of each signal. To compute coherence over time, cross-spectral densities were averaged across 10 data windows (overlap of six windows). Theta coherence was always defined as average coherence between 4 and $12 \mathrm{~Hz}$. For power correlations, the wavelet transforms of the LFP signals in each structure were computed, and theta power over time was obtained by averaging the absolute values of the wavelet transforms in the $4-12 \mathrm{~Hz}$ range. For some analyses, we also computed the partial theta power correlation between two regions that took into account theta power in a third area using the MATLAB function partialcorr.

Phase-locking analyses. LFPs were first filtered in the theta range (4-12 $\mathrm{Hz}$ ) using a zero-phase-delay filter (filter0; provided by K. Harris and G. Buzsáki, New York University, New York, NY) implemented in MATLAB (version 7.6; MathWorks). The filter uses a linear phase function (fir1; MATLAB Signal Processing Toolbox, version 6.9; MathWorks) and compensates for the phase delay by time-shifting the filtered signal. The 
A

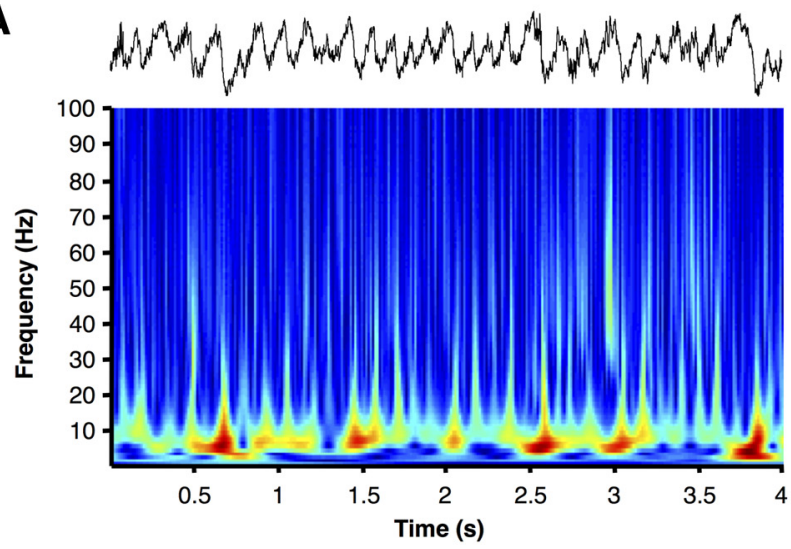

C

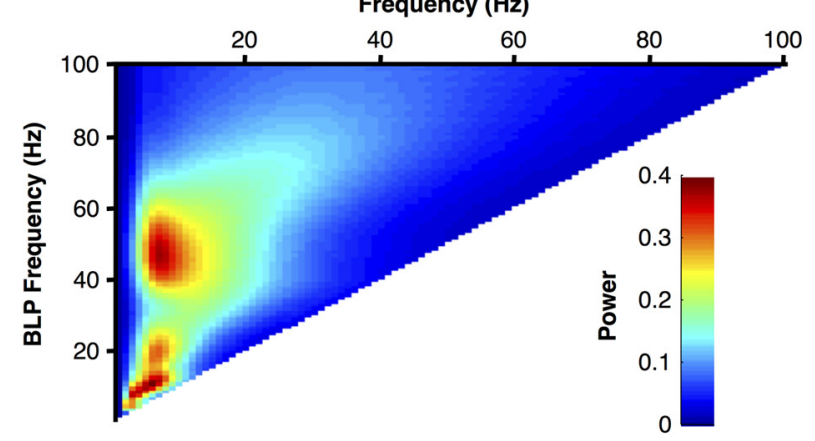

$\mathbf{E}$

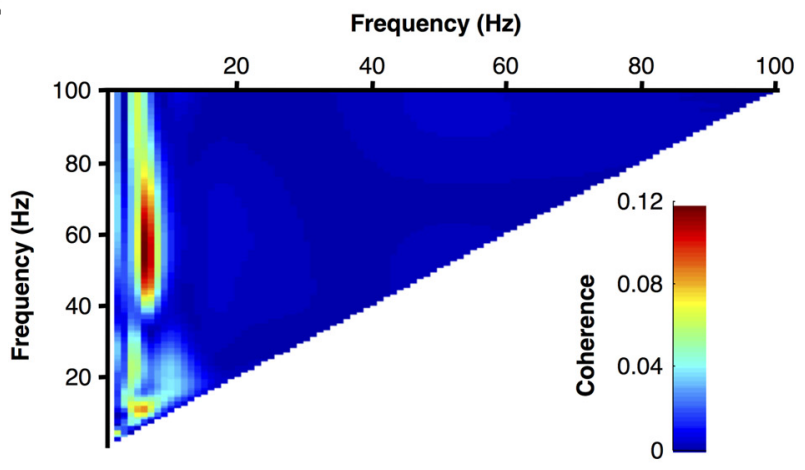

B

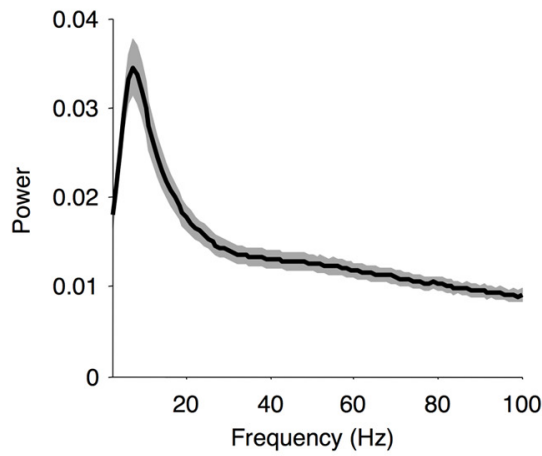

D

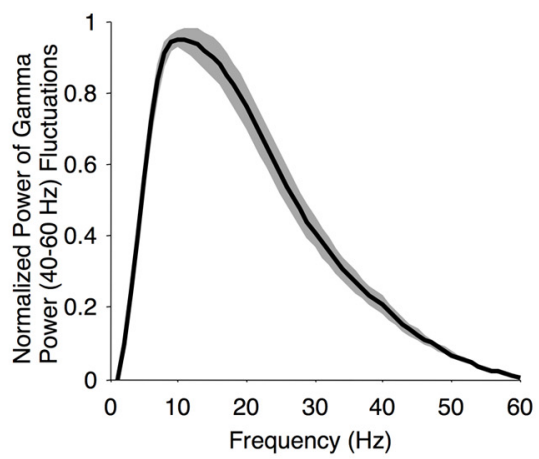

$\mathbf{F}$

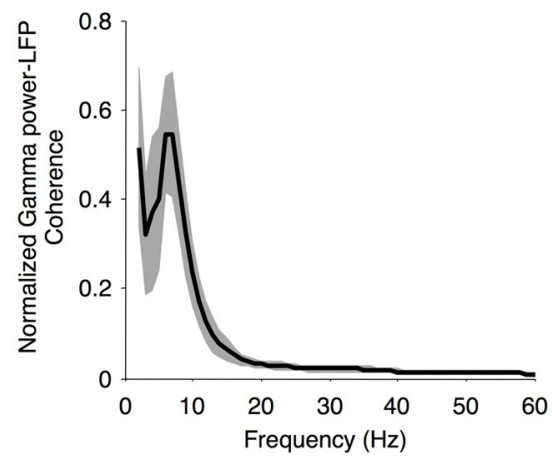

Figure 1. Theta-frequency oscillations in the mPFC. $\boldsymbol{A}$, Example LFP recording from the mPFC (top) and its spectrogram (bottom). Note prominent oscillations in the theta range. $\boldsymbol{B}$, Mean LFP power in the MPFC across animals. C, Power spectra of BLP fluctuations in one animal (see Materials and Methods).Note that the power of gamma ( $40-60 \mathrm{~Hz}$ ) oscillations fluctuates with theta $(4-12 \mathrm{~Hz})$ periodicity. $\boldsymbol{D}$, Mean normalized power spectra of gamma $(40-60 \mathrm{~Hz})$ power fluctuations. $\boldsymbol{E}$, Coherence of BLP fluctuations with the raw LFP in one animal, showing gamma power fluctuating coherently with the raw LFP at theta frequency. $F$, Mean normalized coherence of gamma power fluctuations with the raw LFP across animals. Shaded plots represent the mean \pm SEM over animals ( $n=10$ mice).

phase and amplitude of each sample of the filtered field potential was then computed using the Hilbert transform, and each spike was assigned the phase of its contemporaneous field potential sample. Phase locking was quantified as the circular concentration of the resulting phase distribution, which we defined as the mean resultant length (MRL) of the phase angles. MRL is the sum of unit length vectors representing the phases at which each spike occurred divided by the number of spikes. Therefore, it takes on values between 0 (no phase locking) and 1 (perfect phase locking). The statistical significance of phase locking was assessed using the Rayleigh's test for circular uniformity.

Sample estimates of MRL suffer from a positive bias (that is, they overestimate the population MRL) whose magnitude is highly dependent on sample size. This can cause problems when comparing phase locking across conditions with different numbers of spikes. When com- paring phase locking in the sample and choice phases of the task (see Fig. $3 F$ ), we adopted two measures to limit the effects of this bias on our analysis. First, we only computed phase locking for cells firing at least 50 spikes in both task phases. Second, we subsampled spikes from the task phase with more spikes so that equal numbers of spikes were used for calculating MRL.

Pharmacological inactivation of the $v H P C$. A 26 gauge guide cannula (Plastics One) was implanted in the vHPC using the surgical procedures described above. A single tungsten wire was glued to the side of the cannula so that it extended $1.5 \mathrm{~mm}$ past the cannula tip. Tungsten wires were also bilaterally implanted in the mPFC and dHPC. After recovery from surgery, mice were food deprived to $85 \%$ of their preoperative weight. Saline or muscimol (Tocris Bioscience) dissolved in saline (8.8 mM concentration) was microinfused into the vHPC by back loading into 
A

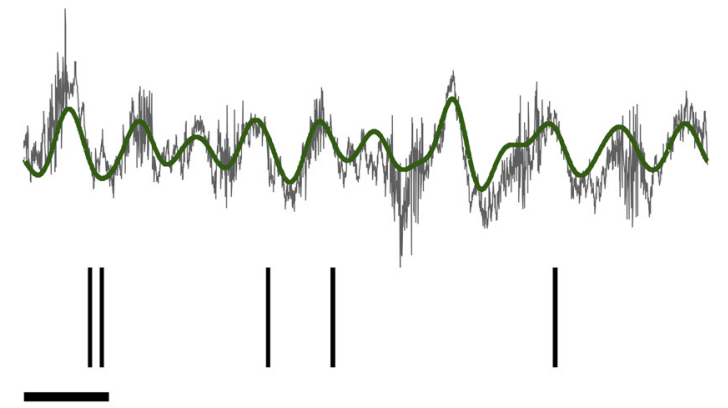

B

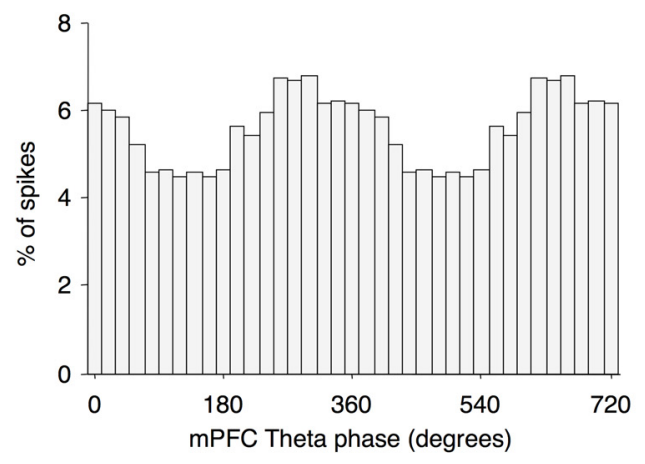

D

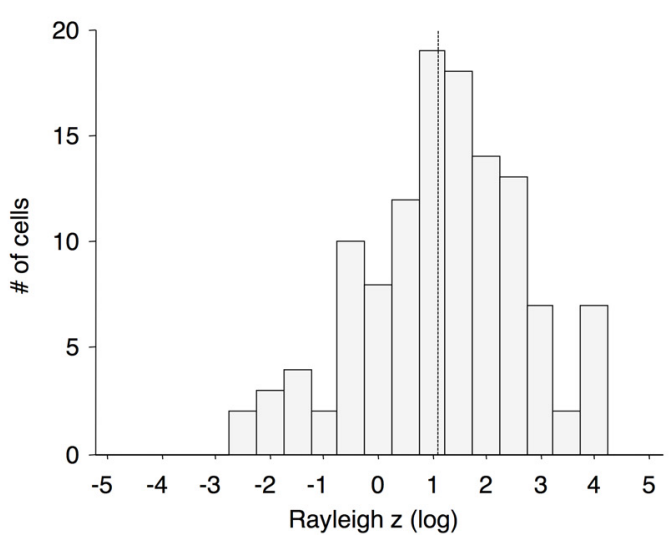

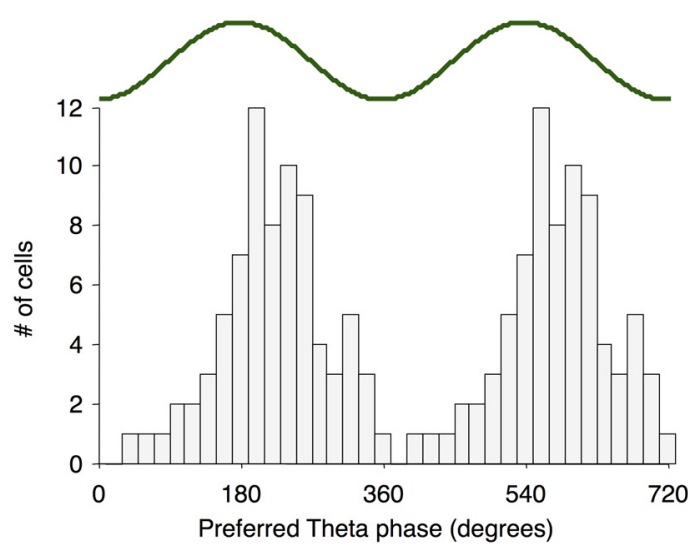

Figure 2. Theta oscillations in the mPFC modulate the spiking of mPFC neurons. $\boldsymbol{A}$, Example of a raw (black) and theta-filtered (4-12 Hz; green) LFP recording from the mPFC and simultaneously recorded spikes from an mPFC neuron (tick marks). Scale bar, $200 \mathrm{~ms}$. $\boldsymbol{B}$, Distribution of theta phases at which the neuron in $\boldsymbol{A}$ fired. Note the robust modulation by theta phase. $\boldsymbol{C}$, Distribution of Rayleigh's $z$ scores, reflecting the strength of phase locking, across mPFC neurons $(n=125)$. The dotted line indicates the significance threshold $(p<0.05)$. $\boldsymbol{D}$, Distribution of theta phases at which phase-locked mPFC neurons $(n=77)$ tended to fire. The green trace is a schematic of the theta cycle.

a 33 gauge infusion cannula and into polyethylene (PE 20) tubing connected to $1.0 \mu \mathrm{l}$ Hamilton microsyringe. The infusion cannula protruded $1 \mathrm{~mm}$ beyond the guide cannula tip. An infusion volume of $0.13 \mu \mathrm{l}$ was delivered using a Harvard 11 Plus syringe pump (Harvard Apparatus) at a rate of $0.13 \mu \mathrm{l} / \mathrm{min}$. The time course of the experiment was as follows. First, saline was injected while the animal was in the home cage. This was followed by a $20-30 \mathrm{~min}$ postinfusion interval, after which neural activity was recorded for $10 \mathrm{~min}$ while animals navigated the T-maze. Next, animals received a second infusion of either saline or muscimol, again followed by a $20-30 \mathrm{~min}$ postinfusion interval and a $10 \mathrm{~min}$ recording as animals navigated the T-maze. Data analysis was restricted to periods when animals were moving at a consistent speed $(5-9 \mathrm{~cm} / \mathrm{s})$. Coherence and power were calculated with methods described above and compared with the Wilcoxon's sign-rank test.

\section{Results}

Theta oscillations in the mPFC reflect local neuronal activity We recorded the LFP and neuronal spiking in the $\mathrm{mPFC}$ of mice performing an SWM task (see Materials and Methods). During all phases of the task, oscillations in the LFP were most pronounced in the theta-frequency range (Fig. $1 A, B ; 7.3 \pm 0.15 \mathrm{~Hz}$ ). Because the LFP may reflect electrical activity volume conducted from areas distant to the recording site, we sought evidence that the observed theta-frequency oscillations reflected local neural activity. Gamma oscillations, which tend to be highly localized spatially (Sirota et al., 2008), were also observable in our mPFC recordings. Therefore, we asked whether gamma power fluctuated with theta periodicity. For this analysis, we first extracted estimates of BLP over time with center frequencies between 1 and $100 \mathrm{~Hz}$ (see Materials and Methods). We then subjected the BLP time series to spectral analysis to reveal any periodicity in the power fluctuations within each frequency band. This analysis revealed that LFP power in the gamma range (40-60 $\mathrm{Hz}$ ) fluctuated with a periodicity that was similar to the observed $\mathrm{mPFC}$ theta oscillations (Fig. 1C,D). These fluctuations in gamma power were also coherent with the raw LFP in the theta range (Fig. $1 E, F)$, strongly arguing that the recorded theta-frequency oscillations are indeed of local origin. To further confirm this, we next asked whether locally recorded theta oscillations were reflected in the spiking of $\mathrm{mPFC}$ neurons. We found that the majority of $\mathrm{mPFC}$ neurons modulated their firing as a function of the phase of the theta oscillation (77 of 125 neurons; $p<0.05$, Rayleigh's test; Fig. $2 A-C$ ), with most neurons maximally active during the descending phase of the oscillation (Fig. 2D). Together, these results suggest that theta oscillations in the mPFC reflect local activity and not volume conduction from distant regions.

\section{Theta oscillations in the MPFC are modulated during SWM}

If theta oscillations are important for SWM, their strength should be modulated by the mnemonic demands of the task and correlate with the animals' behavioral performance. To examine this, we compared neural activity in the "sample" and "choice" phases of the discrete trial T-maze task (Fig. 3A; see Materials and Methods). Although the overt behavior is the same in these two task phases, it is only in the choice phase that the animals must use 
A
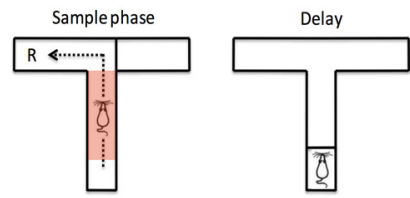

C

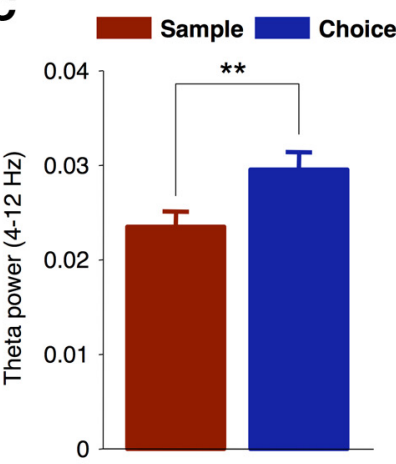

B

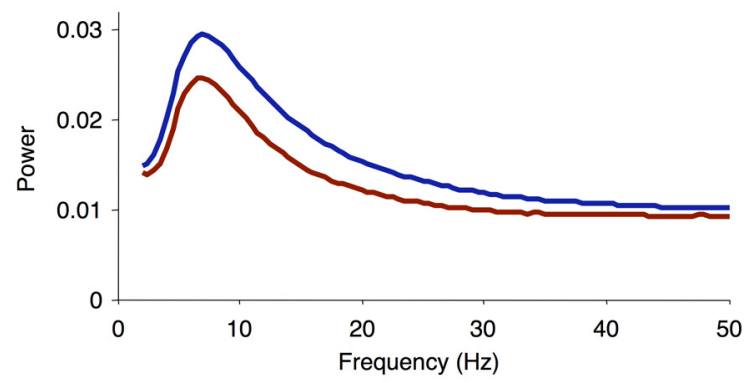

D

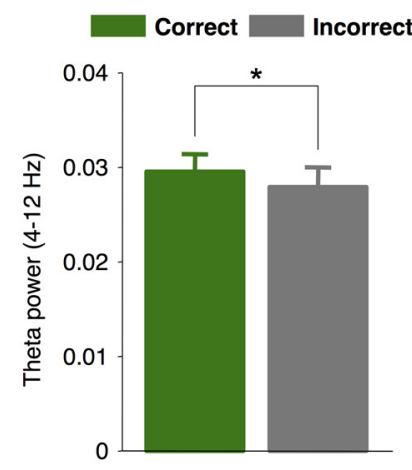

E

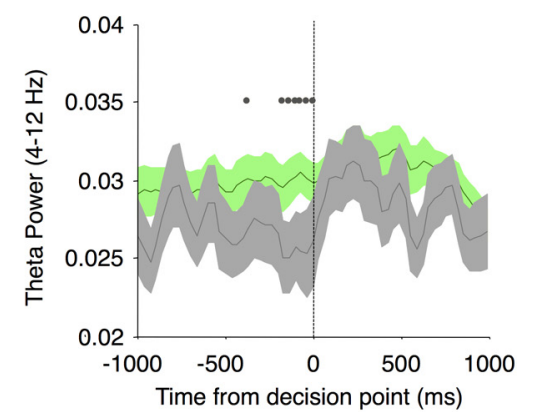

F

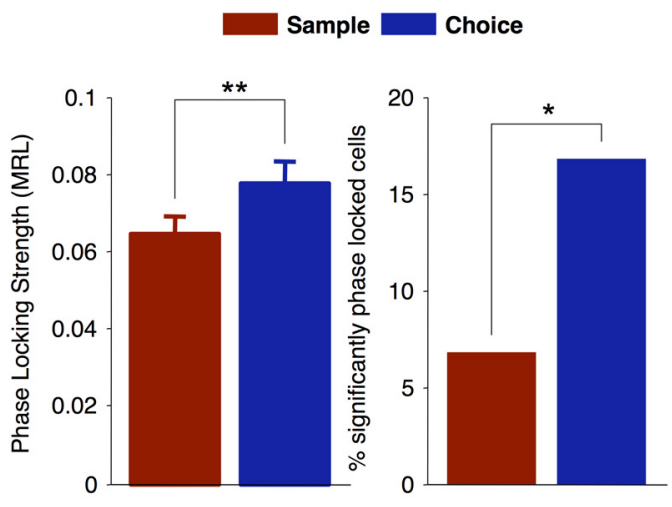

G

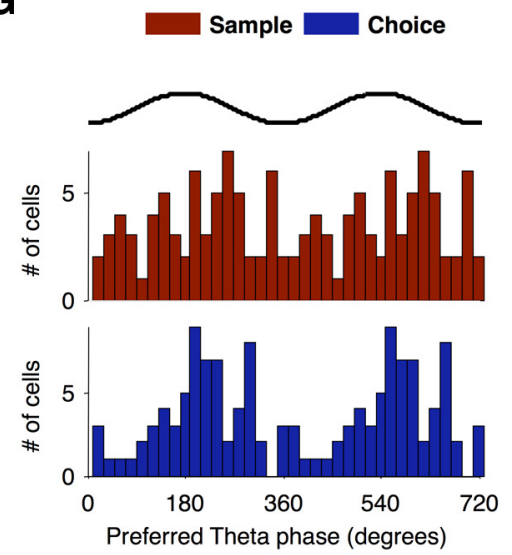

Figure 3. $\mathrm{mPFC}$ theta oscillations are modulated by SWM behavior. $A$, Schematic of the discrete trial T-maze task. Working memory is required during the choice phase (blue) but not the sample phase (red; for details, see Materials and Methods). B, Examples of power spectra from sample and choice phases during one session. C, Mean theta power during sample and choice phases. $\boldsymbol{D}$, Mean theta power during the choice phase of correct (green) and incorrect (gray) trials. $E$, Mean theta power during the choice phase of correct (green) and incorrect (gray) trials as a function of time before reaching the intersection of the T-maze. Note that theta power on incorrect trials decreases shortly before the animals reach the intersection, when they must choose to go left or right. Filled squares indicate significant differences between correct and incorrect trials $(p<0.05$; Wilcoxon's sign-rank test). $\boldsymbol{F}$, Strength of phase locking (left; $n=65$ neurons) and percentage of phase-locked neurons (right; $n=119$ ) in the sample and choice phases. G, Distribution of theta phase preference across mPFC neurons in the two task phases. Phase preference becomes more concentrated in the choice phase, suggesting increased synchrony. Unless otherwise stated, error bars represent the mean \pm SEM over animals $\left(n=10\right.$ mice). ${ }^{*} p<0.05,{ }^{* *} p<0.01$.

their memory of which arm they visited previously to guide their decision. Any differences in neural activity during these two epochs can therefore yield insights into the neural processes underlying SWM. Indeed, the amplitude of theta oscillations in the mPFC during the choice phase was higher than in the sample phase (Fig. $3 B, C ; p<0.01$, Wilcoxon's signed-rank test). In contrast, incorrect choices were associated with a decreased amplitude of theta oscillations compared with correct choices (Fig. 3D; $p<0.05$, Wilcoxon's signed-rank test). Additional analysis revealed that theta power during correct and incorrect trials became significantly different shortly before the animals reached the end of the center arm of the maze, at which point they had to decide whether to enter the left or the right goal arm (Fig. $3 E ; p<$
0.01, Wilcoxon's signed-rank test). Consistent with the LFP results, the strength of phase locking of mPFC neurons to the local theta oscillation ( $p<0.01$, Wilcoxon's signed-rank test; 65 neurons), as well as the proportion of significantly phase-locked cells $(p<0.05$, Fisher's exact test) increased during the choice phase (Fig. $3 F$ ). Furthermore, the distribution of theta phases at which prefrontal neurons preferentially fired became significantly concentrated in the choice phase (Fig. 3G; $p<0.05$, bootstrap test of MRL of preferred phases during sample and choice), suggesting an increase in theta-mediated synchrony among mPFC neurons.

Although we observed an increase in theta synchrony in the $\mathrm{mPFC}$, it is possible that this is attributable to greater neural activity in the choice phase. Alternatively, it is possible that the 
A

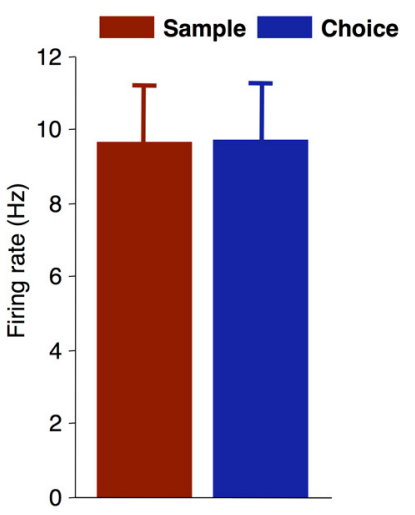

C

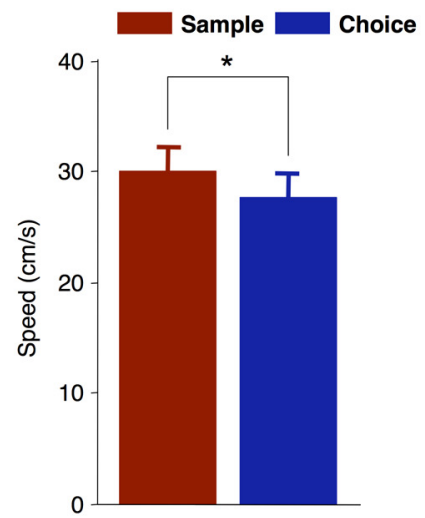

B

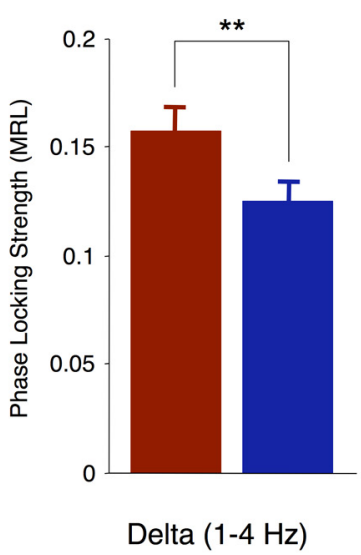

D

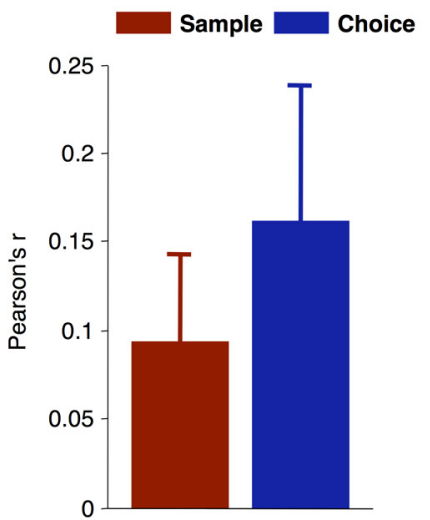

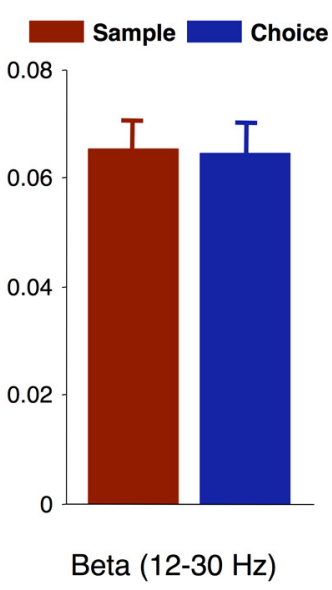

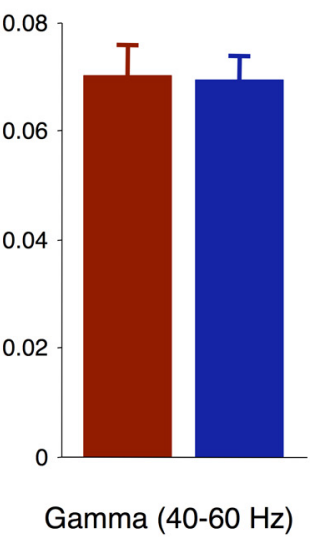

E

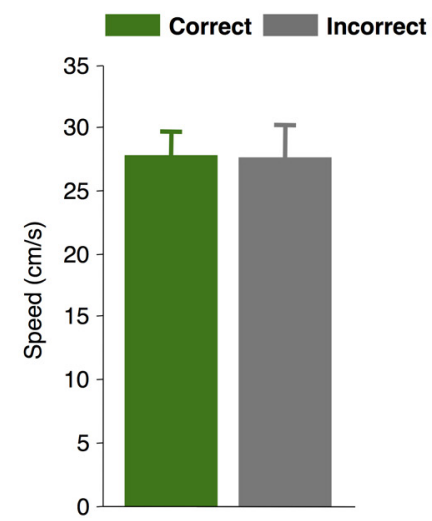

Figure 4. $\quad \mathrm{mPFC}$ theta oscillations are selectively enhanced during working memory and are not attributable to differences in running speed. $\boldsymbol{A}$, Firing rate of $\mathrm{mPFC}$ neurons during sample and choice phases. $\boldsymbol{B}$, Phase locking of mPFC neurons to delta, beta, and gamma oscillations during sample and choice. Note the lack of increase in phase locking during the choice phase. $\boldsymbol{C}$, Running speed of animals during the sample and choice phases. $\boldsymbol{D}$, Correlation between running speed and theta power for the two task phases. Note the absence of a significant correlation. $\boldsymbol{E}$, Running speed during choice phases of correct and incorrect trials. Error bars represent mean \pm SEM across animals $(n=10) .{ }^{*} p<0.05,{ }^{* *} p<0.01$.

increase in synchrony is not specific to the theta range but rather reflects a general increase in synchrony at all frequencies. To examine these possibilities, we first compared the firing rates of neurons in the sample and choice phase and did not find them to be significantly different (Fig. $4 A ; p>0.05$, Wilcoxon's sign-rank test). Next, we examined phase locking of mPFC neurons to delta $(1-4 \mathrm{~Hz})$, beta $(12-30 \mathrm{~Hz})$, and gamma $(40-60 \mathrm{~Hz})$ oscillations. In contrast to the increase in theta phase locking, we found that phase locking to delta oscillations actually decreased during the choice phase (Fig. $4 B ; p<0.01$, Wilcoxon's sign-rank test), whereas phase locking to beta and gamma oscillations did not change (Fig. $4 B ; p>0.05$, Wilcoxon's sign-rank test). Therefore, these results suggest a selective increase of mPFC theta synchrony during the SWM task. Another possible explanation of our results is that the running speed of the animals differed in the two task phases. In the hippocampus, the magnitude of theta oscillations is positively correlated with the running speed, and therefore, this could influence our results. We found that running speed was actually slightly lower during the choice phase (Fig. 4C; 27.6 vs 30.0 $\mathrm{cm} / \mathrm{s}, p<0.05$, Wilcoxon's sign-rank test). To examine whether this could affect our results, we computed the correlation between running speed and theta power on a trial-bytrial basis, separately for each animal during the sample and choice phases. Although correlations tended to be positive, the distributions for both sample and choice phases were not significantly different from zero (Fig. $4 D ; p>0.05$, Wilcoxon's sign-rank test), suggesting a weak relationship between running speed and theta power in the mPFC. Also note that a positive correlation would cause greater theta power in the sample phase, which is the opposite of what we observed. Finally, we compared running speed during choice phase runs in correct and incorrect trials but did not find them to be significantly different (Fig. $4 E$; $p>0.05$, Wilcoxon's sign-rank test). Therefore, we conclude that differences in overall neural activity or running speed are unlikely to account for our results.

\section{Theta oscillations in the vHPC but not the dHPC are modulated during SWM}

A major source of inputs to the $\mathrm{MPFC}$ comes from the hippocampus, which is known to generate robust theta oscillations (Buzsáki, 2002). Although most previous studies have focused on theta oscillations in the dHPC, this subregion of the hippocampus only projects indirectly to the mPFC. However, the vHPC gives rise to monosynaptic excitatory projections to the mPFC (Dégenètais et al., 2003; Hoover and Vertes 2007). Therefore, we asked how theta oscillations in the vHPC and $\mathrm{dHPC}$ compare with those in the $\mathrm{MPFC}$ and whether they 
A

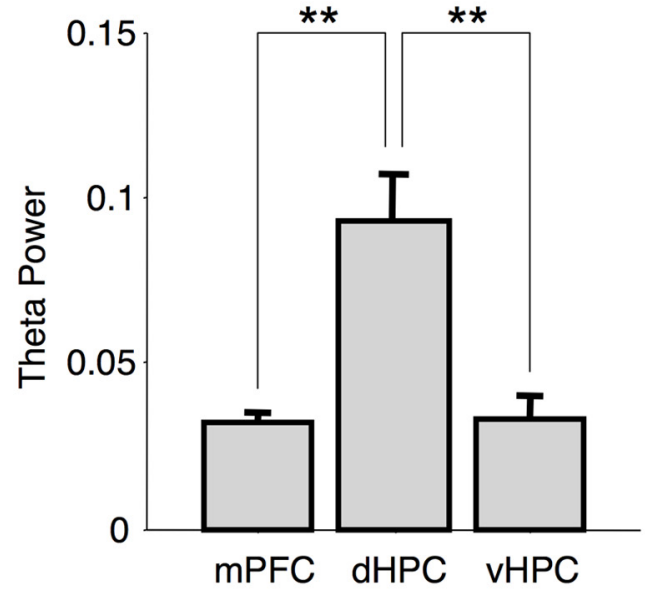

Dorsal Hippocampus

C

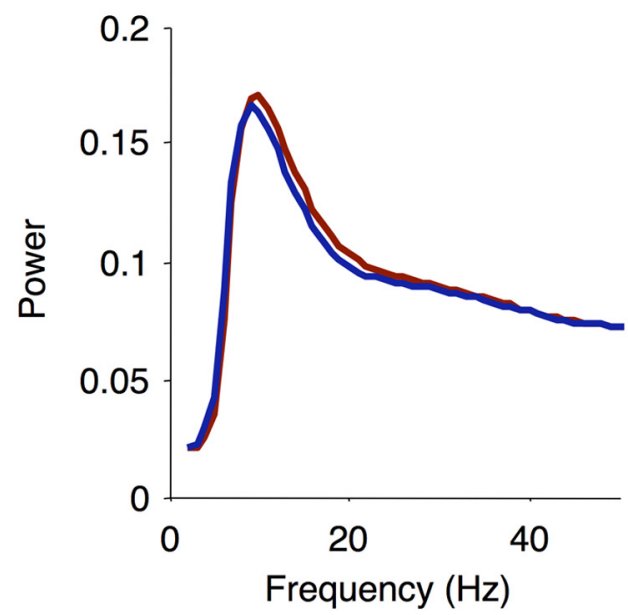

E

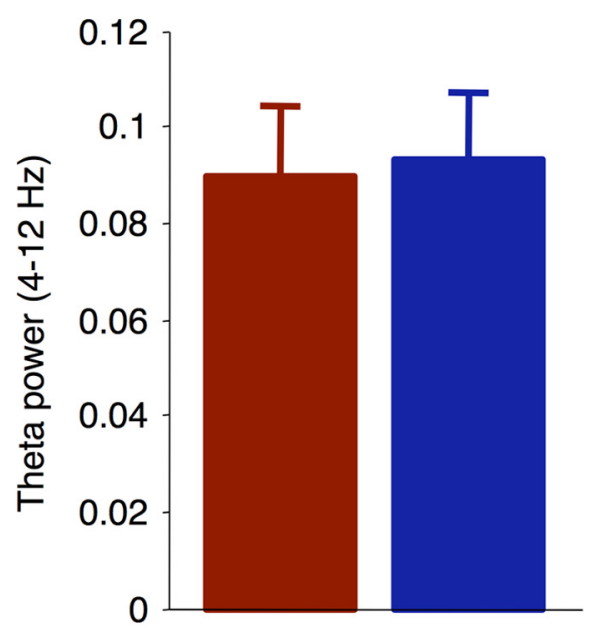

B

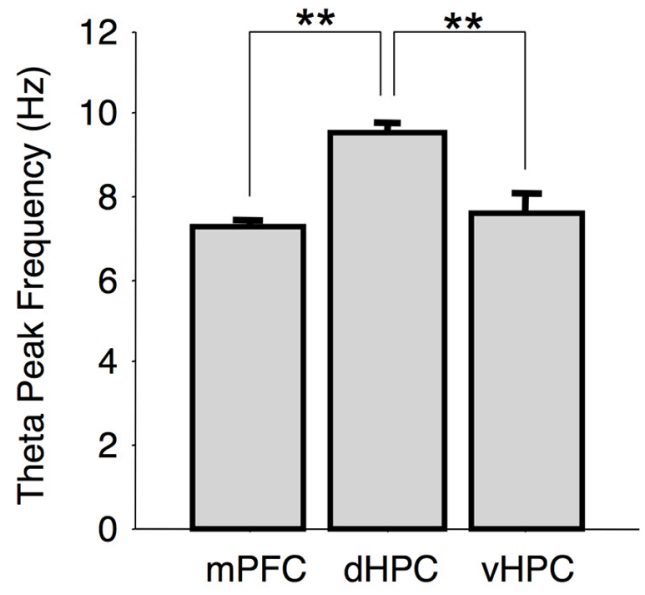

Ventral Hippocampus

D

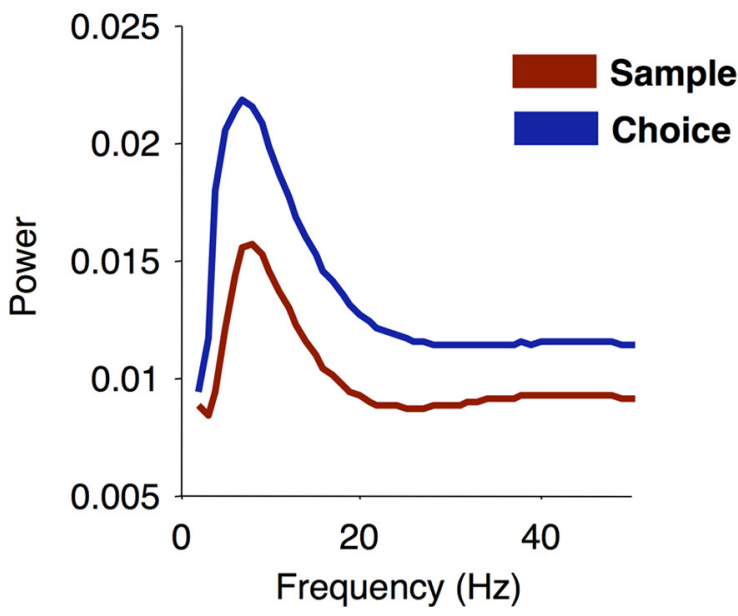

$\mathbf{F}$

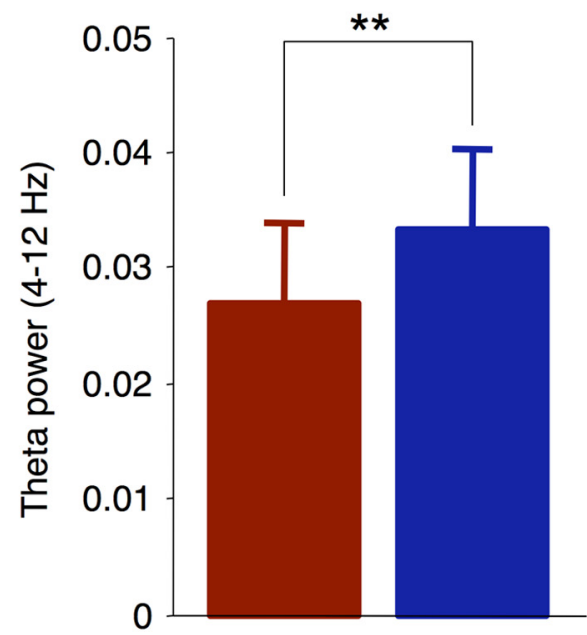

Figure 5. Modulation of hippocampal theta oscillations by SWM. $\boldsymbol{A}, \boldsymbol{B}$, Power $(\boldsymbol{A})$ and frequency $(\boldsymbol{B})$ of theta oscillations recorded in the mPFC, dHPC, and vHPC. Note the similarity of $\mathrm{mPFC}$ and vHPC theta oscillations. $\boldsymbol{C}, \boldsymbol{D}$, Example power spectra of LFPs recorded in the $\mathrm{dHPC}(\boldsymbol{C})$ and $\mathrm{vHPC}(\boldsymbol{D})$ of one animal during sample and choice phases. $\boldsymbol{E}$, $\boldsymbol{F}$, Theta power in dHPC $(\boldsymbol{E})$ and $\operatorname{VHPC}(\boldsymbol{F})$ during sample and choice phases. Note that theta power increases in the choice phase only in the vHPC. Error bars represent mean \pm SEM across animals $(n=5-10)$. ${ }^{* *} p<0.01$. 


\section{A}

dHPC-mPFC

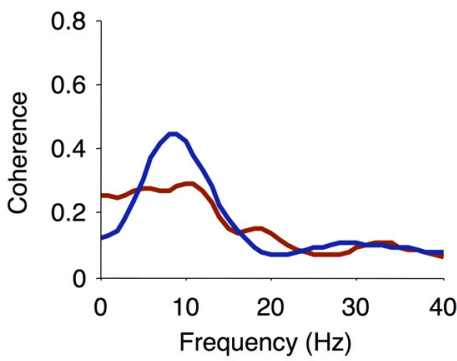

B

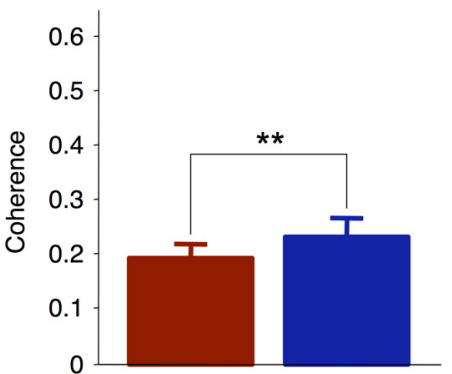

C
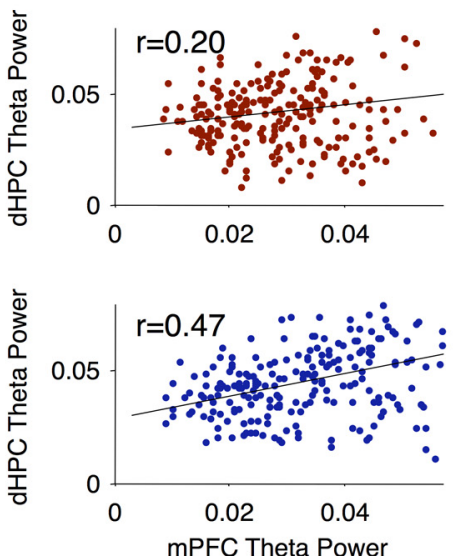

D

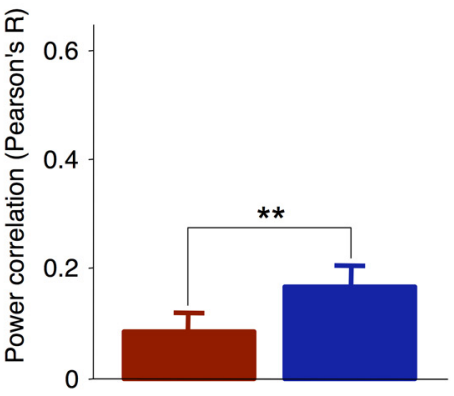

vHPC-mPFC
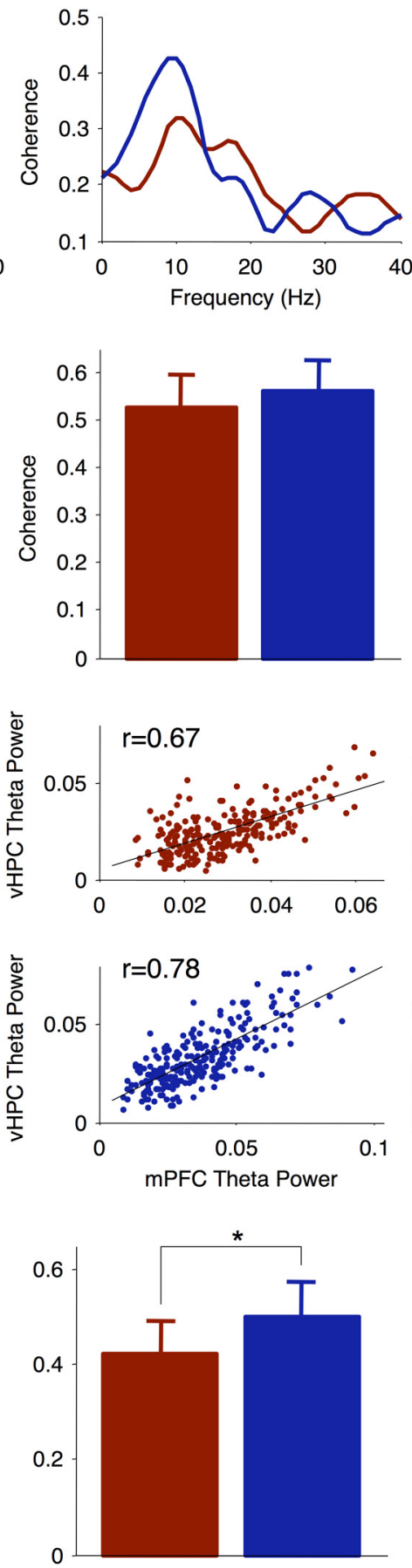

dHPC-vHPC
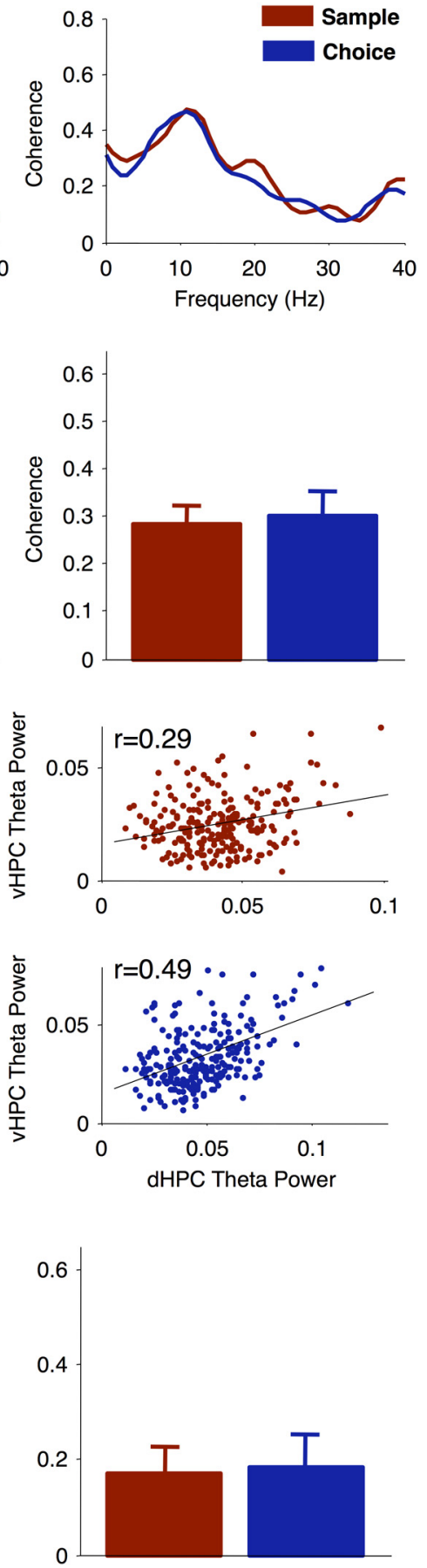

Figure 6. Hippocampal-prefrontal theta synchrony is modulated by the SWM task. The columns illustrates synchrony between dHPC and mPFC (left), vHPC and mPFC (middle), and dHPC and $\operatorname{VHPC}($ right). $\boldsymbol{A}$, Examples of coherence during sample and choice phases in individual animals. $\boldsymbol{B}$, Mean theta coherence during sample and choice phases. $\boldsymbol{C}$, Examples of theta power correlations during sample and choice phases in individual animals. $\boldsymbol{D}$, Mean theta power correlations during sample and choice phases. Note that theta synchrony increases between both hippocampal subregions and the mPFC, whereas intrahippocampal synchrony remains unchanged. Error bars represent mean \pm SEM across animals $(n=13) .{ }^{*} p<0.05,{ }^{* *} p<0.01$.

are also modulated by the SWM task. Theta oscillations in the dHPC were both larger in amplitude and of a higher frequency than in either the vHPC or mPFC (Fig. $5 A, B ; p<0.01$, Wilcoxon's signed-rank test), consistent with similar observations under other behavioral conditions (Adhikari et al., 2010; Royer et al., 2010). However, theta oscillations did not differ between the vHPC or MPFC in terms of either power or fre- quency ( $p>0.05$, Wilcoxon's signed-rank test). Furthermore, we found that theta oscillations in the vHPC, but not the $\mathrm{dHPC}$, increased in strength during the choice phase (Fig. $5 C-F)$. Thus, theta oscillations in the vHPC resemble mPFC theta oscillations, both qualitatively and in terms of their modulation by the SWM task, possibly reflecting the direct connections between the two areas. 
A

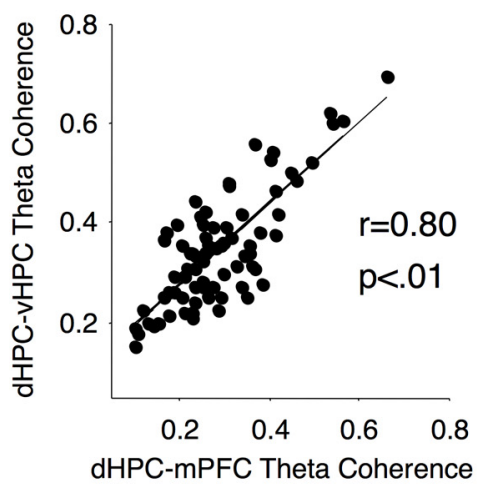

B

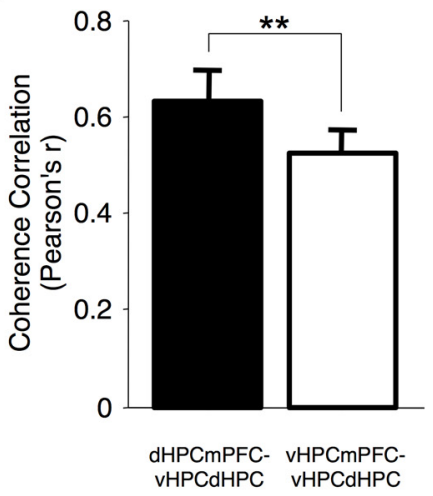

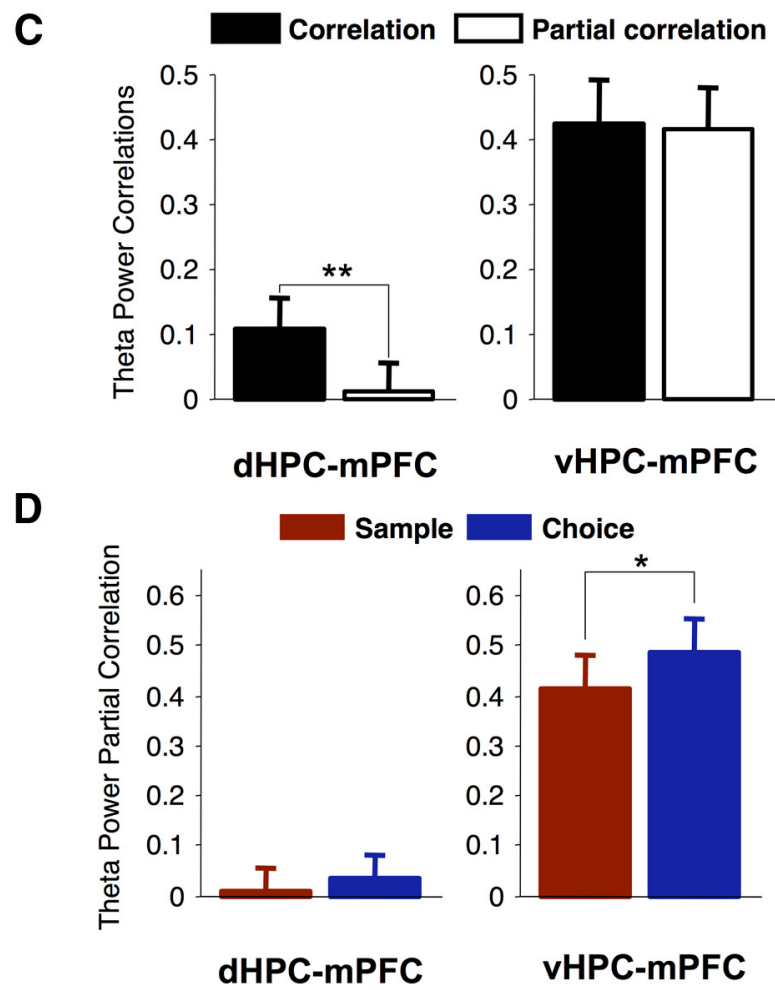

Figure 7. Hippocampal-prefrontal synchrony is correlated with ventral hippocampal activity. $\boldsymbol{A}$, Positive correlation between dHPC-mPFC and dHPC-vHPC coherence in an example animal. $\boldsymbol{B}$, Mean correlations between $\mathrm{dHPC}-\mathrm{mPFC}$, vHPC-mPFC, and $\mathrm{dHPC}-\mathrm{vHPC}$ theta coherence $C$, Mean theta power partial correlations. Note that partial correlations are lower for $\mathrm{AHPC}-\mathrm{mPFC}$ but not vHPC $-\mathrm{mPFC}$. $D$, Partial correlations during sample and choice phases. Error bars represent mean \pm SEM across animals $(n=$ 13). ${ }^{*} p<0.05,{ }^{* *} p<0.01$.

The vHPC mediates hippocampal-prefrontal theta synchrony These results raise the possibility that theta oscillations in the mPFC, and their modulation by SWM, might be driven by theta activity in the hippocampus, perhaps via the vHPC. If so, one would expect theta-frequency synchrony between both hippocampal subregions and the mPFC to be modulated by the SWM task. To address this issue, we measured both coherence and power correlations between the mPFC and both vHPC and $\mathrm{dHPC}$. Coherence in the theta-frequency range increased during the choice phase between the dHPC and mPFC (Fig. $6 A, B ; p<$ 0.01 , Wilcoxon's signed-rank test) but not between the vHPC and $\mathrm{mPFC}$ (Fig. $6 A, B ; p>0.05$, Wilcoxon's signed-rank test). However, using power correlations as a measure of synchrony revealed an increase in theta power correlations between the mPFC and both the dHPC $(p<0.01)$ and vHPC $(p<0.05$,
Wilcoxon's signed-rank test) during the choice phase (Fig. 6C,D). In contrast, theta synchrony did not increase between the dHPC and vHPC, measured using either coherence or power correlations (Fig. $6 A-D ; p>0.05$, Wilcoxon's signed-rank test).

Although both the dHPC and vHPC display theta synchrony with the MPFC, only the $\mathrm{vHPC}$ projects directly to the $\mathrm{MPFC}$, allowing for the possibility that hippocampal-mPFC theta synchrony is mediated by the vHPC. Consistent with this, we found that periods of high $\mathrm{dH}$ PC-mPFC theta coherence were associated with high dHPC-vHPC theta coherence (Fig. $7 A, B$ ), suggesting the possibility that, for the dHPC to synchronize with the $\mathrm{mPFC}$, it must also synchronize with the vHPC. Notably, vHPC-mPFC coherence was less correlated with dHPC-vHPC coherence $(p<$ 0.01 , Wilcoxon's sign-rank test; Fig. $7 B$ ), suggesting that the ability of the vHPC to synchronize with the mPFC did not depend on its ability to synchronize with the dHPC. To further assess the contribution of vHPC to hippocampal-prefrontal synchrony, we calculated partial correlations between dHPC and mPFC theta power, taking into account their correlations with the vHPC (Fig. 7C, left). When compared with the simple correlation (i.e., without taking into account the effects of vHPC activity), the partial correlation of $\mathrm{dHPC}$ and $\mathrm{mPFC}$ theta power was significantly lower ( $p=$ 0.01 , Wilcoxon's signed-rank test). Importantly, however, the vHPC-mPFC theta power partial correlation (i.e., taking into account activity in dHPC) was not significantly different from its simple correlation (Fig. $7 C$, right; $p>0.05$, Wilcoxon's signedrank test). We also reexamined the modulation of hippocampal-prefrontal synchrony by SWM using the partial correlation method. This revealed that dHPC-mPFC theta power correlations did not increase in the choice phase when vHPC activity was taken into account (Fig. $7 D ; p>0.05$, Wilcoxon's signed-rank test), whereas the increase in vHPC-mPFC power correlations remained significant after taking into account dHPC activity (Fig. 7D; $p<0.05$, Wilcoxon's signedrank test).

Together, these results support the hypothesis that hippocampal-prefrontal theta synchrony is primarily mediated by the vHPC. This hypothesis predicts that inactivating the vHPC should disrupt hippocampal-prefrontal synchrony. To test this prediction, we recorded LFPs from the MPFC, dHPC, and vHPC before and after infusion of the $\mathrm{GABA}_{\mathrm{A}}$ receptor agonist muscimol into the vHPC (see Materials and Methods). As an initial test of the action and time course of muscimol, we injected one animal with muscimol in the vHPC and observed a decrease in $\mathrm{dH}$ PC-mPFC coherence $\sim 16$ min after infusion (Fig. $8 A$ ). We then 


\section{A}

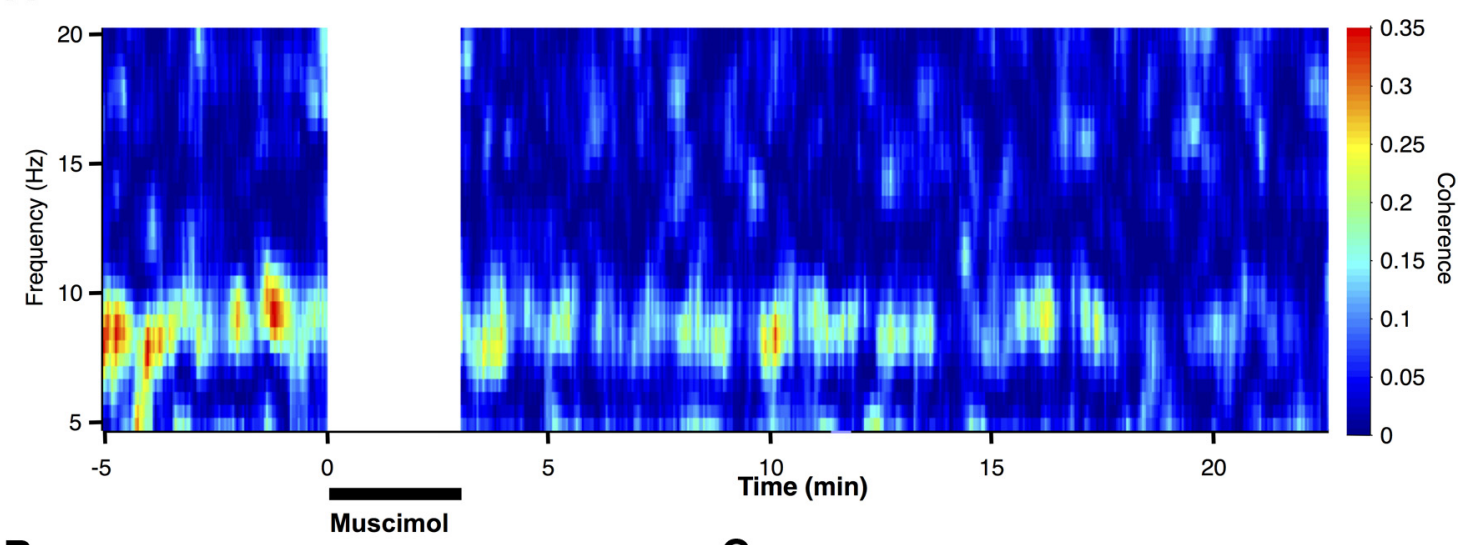

B
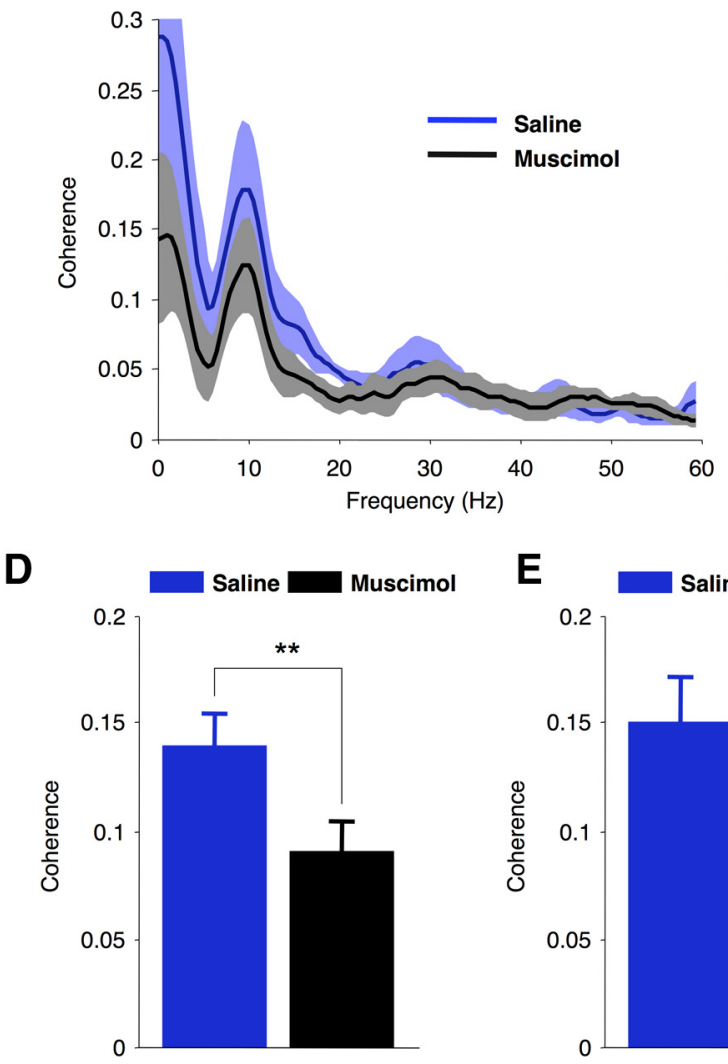

E

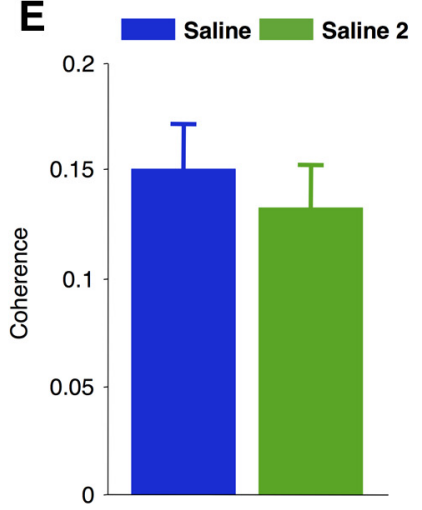

C

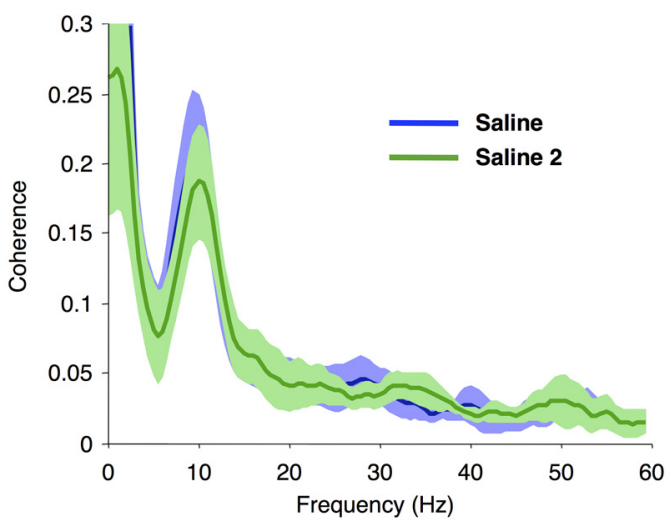

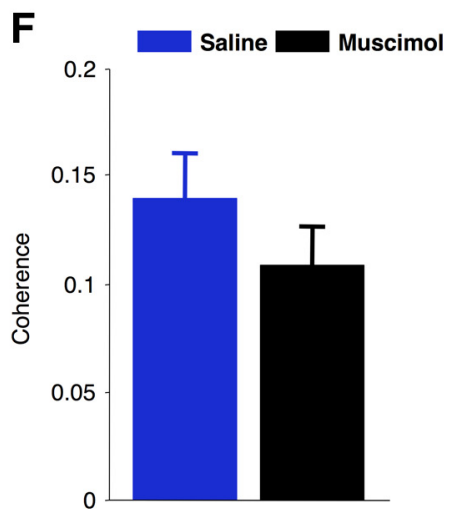

Figure 8. The vHPC is required for hippocampal-prefrontal theta synchrony. $\boldsymbol{A}, \mathrm{dHPC}-\mathrm{mPFC}$ coherence over time before and after muscimol infusion into vHPC in one animal. $\boldsymbol{B}$, Mean $\mathrm{dHPC}-\mathrm{mPFC}$ coherence after muscimol or saline infusions into vHPC. Note the decrease in theta coherence compared with saline infusion. $C$, Mean coherence after a second infusion of saline. $\boldsymbol{D}$, Mean coherence between the dHPC and mPFC after infusions of saline and then muscimol into the ipsilateral vHPC. $E$, Mean coherence between the $\mathrm{dHPC}$ and $\mathrm{mPFC}$ after infusions of saline and then a second saline infusion (saline 2) into the ipsilateral vHPC.F, Mean coherence between the $\mathrm{dHPC}$ and $\mathrm{mPFC}$ after infusions of saline and then muscimol into the contralateral vHPC. Note the selective reduction of theta coherence after inactivation of the VHPC ipsilateral to the recording sites. Error bars represent mean \pm SEM across animals $(n=10) .{ }^{* *} p=0.01$.

performed a controlled experiment to compare dHPC-mPFC coherence after infusion of saline and muscimol. We found that vHPC inactivation decreased AHPC-mPFC theta coherence (Fig. $8 B, D ; p=0.01$, Wilcoxon's sign-rank test), confirming the role of vHPC in hippocampal-prefrontal synchrony. To control for possible effects of multiple injections, we also injected saline twice into the vHPC. However, this had no effect on hippocampal-prefrontal synchrony (Fig. 8C,E; $p>0.05$ ). Because we had implanted electrodes bilaterally into each region, we were able to assess the effect of unilateral inactivation of the vHPC on dHPC$\mathrm{mPFC}$ coherence on the contralateral side. Because of the ipsilateral connectivity of the hippocampal-prefrontal circuit, this served as an important within-animal control. Importantly, we did not observe a significant change in $\mathrm{dHPC}-\mathrm{mPFC}$ coherence contralateral to the vHPC in which muscimol was infused (Fig. $8 F ; p>0.05$, Wilcoxon's sign-rank test).

We next examined the effect of vHPC inactivation on oscillations within the three structures. We observed a significant decrease in vHPC gamma-frequency $(40-60 \mathrm{~Hz})$ power after muscimol infusions (Fig. $9 A, B ; p<0.01$, Wilcoxon's sign-rank test), confirming the efficacy of the inactivation. However, although vHPC theta power was lower after muscimol infusion, this effect did not reach statistical significance (Fig. 9B; $p=0.08$, Wilcoxon's sign-rank test). This suggests that the theta oscillation recorded in the vHPC may 
A

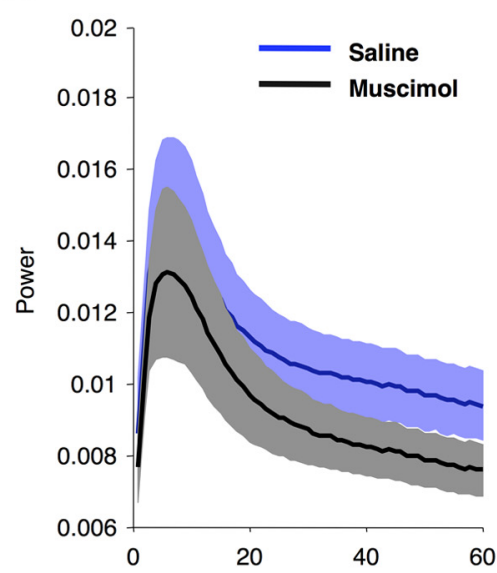

B

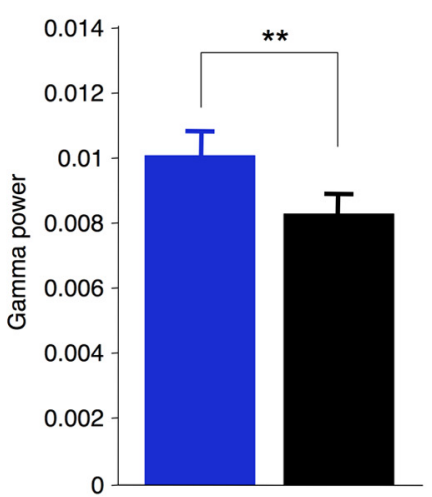

C

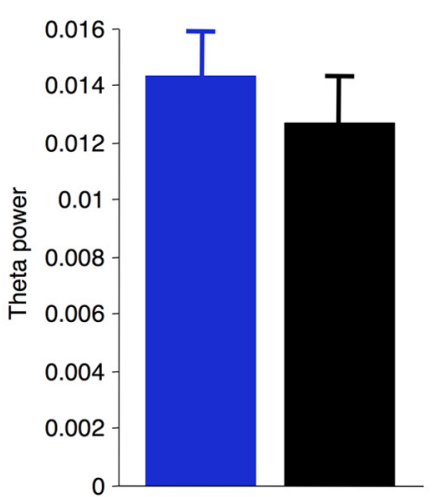

dHPC
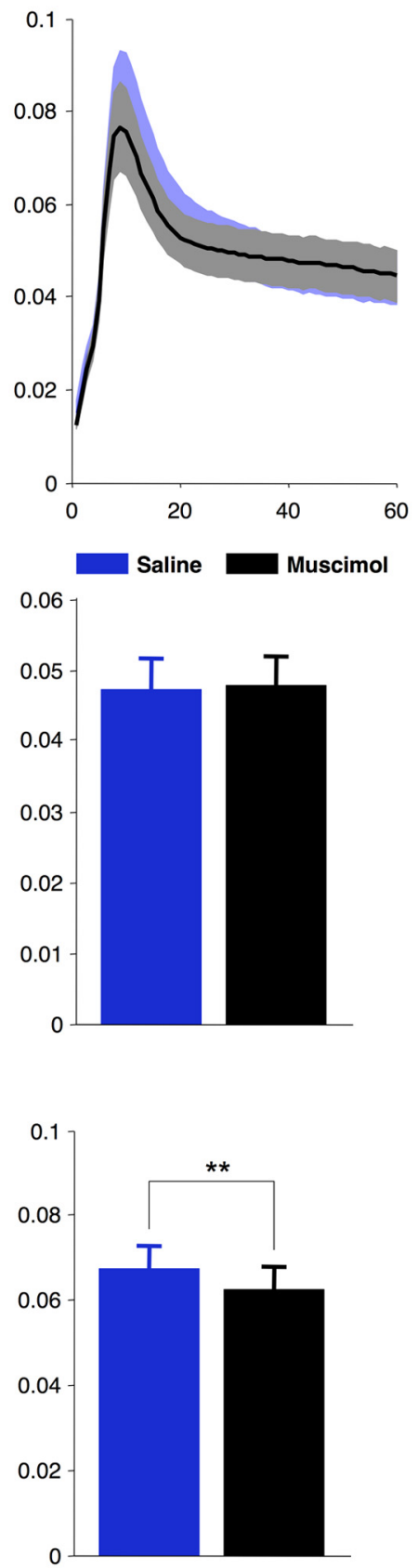

MPFC
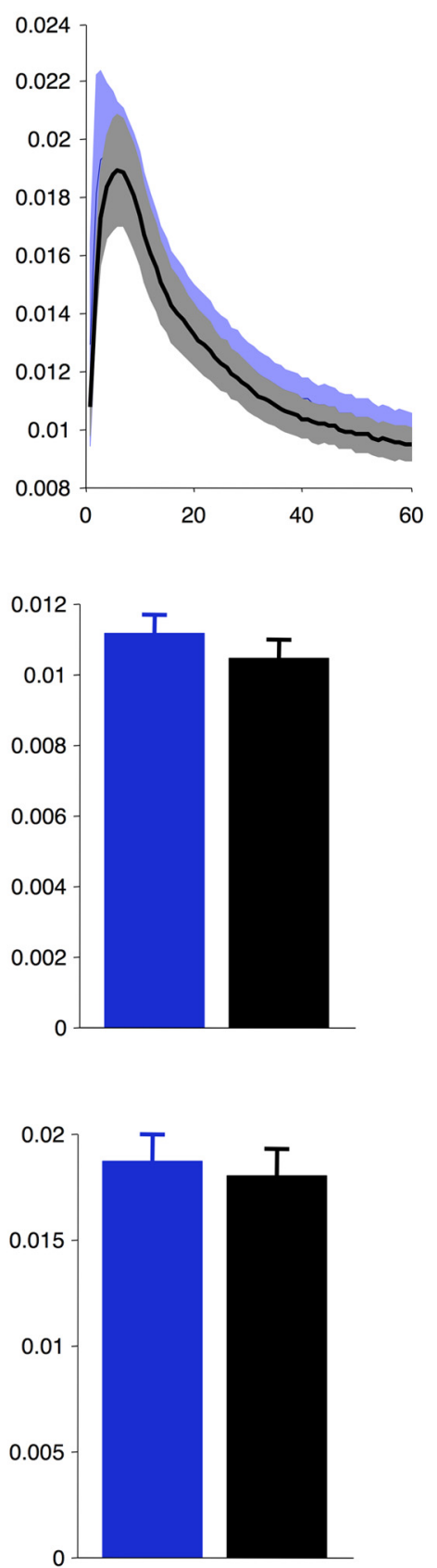

Figure 9. Effect of vHPC inactivation on theta and gamma oscillations in the hippocampus and prefrontal cortex. $A$, Power spectra of vHPC, dHPC, and mPFC after saline and muscimol infusions into the vHPC. $\boldsymbol{B}, \boldsymbol{C}$, Mean gamma $(\boldsymbol{B})$ and theta $(\boldsymbol{C})$ power in vHPC, dHPC, and $\mathrm{mPFC}$ after saline and muscimol infusions into the vHPC. Error bars represent mean \pm SEM across animals $(n=12$ mice). ${ }^{* *} p<0.01$.

arise in larger part from its synaptic inputs rather than local circuit activity. In the dHPC, there was a small but statistically significant decrease in theta power after muscimol infusions but no change in gamma power (Fig. $9 A, C$; $p<0.01$ and $p>0.05$ for theta and gamma power, respectively, Wilcoxon's sign-rank test). We were concerned that the change in hippocampal-prefrontal synchrony after vHPC inactivation could be secondary to the decrease in dHPC theta power. Therefore, we asked whether the effects of muscimol on dHPC-mPFC coherence were correlated with its effects on dHPC theta power. However, we found no evidence of such a relationship (Fig. 10; Pearson's $r=0.124, p>0.05$ ), suggesting that the change in dHPC-mPFC coherence could not be explained by the decrease in
dHPC power alone. Finally, we found that neither mPFC theta (Fig. $9 A, B$ ) nor gamma power (Fig. $9 A, C$ ) was affected by vHPC inactivation ( $p>0.05$, Wilcoxon's sign-rank test). This suggests that the vHPC may not play a major role in generating theta oscillations in the mPFC. Rather, our data suggest that the vHPC is critical for coordinating theta oscillations in the $\mathrm{MPFC}$ with theta oscillations in the hippocampus.

\section{Discussion}

Here we examined theta oscillations recorded from the mPFC of mice performing a SWM task. We found that theta oscillations were the most prominent feature of the MPFC LFP and 


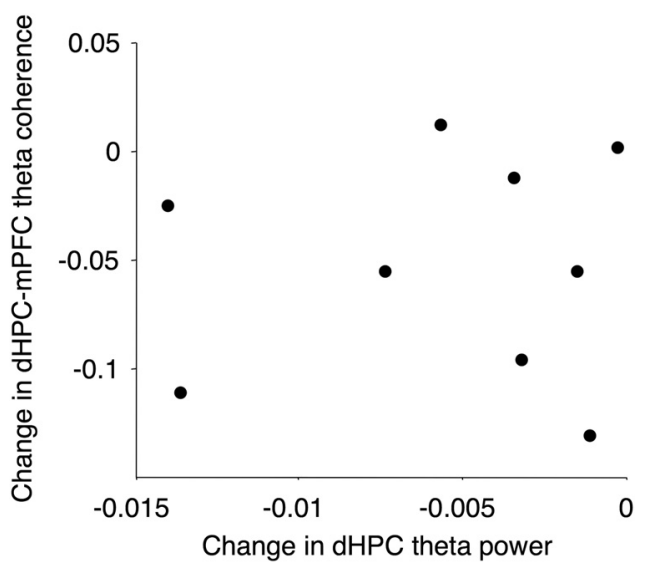

Figure 10. The decrease in $\mathrm{dHPC}-\mathrm{mPFC}$ theta coherence after vHPC inactivation cannot be explained by a decrease in $\mathrm{HHPC}$ theta power. The scatter plot shows, for each animal, the change in $\mathrm{AHPC}-\mathrm{mPFC}$ theta coherence against the change in $\mathrm{AHPC}$ theta power after vHPC muscimol infusions. Negative values indicate lower power or coherence after muscimol infusions. Note the absence of a correlation between the two variables (Pearson's $r=$ $0.12, p>0.05)$.

that these oscillations were synchronized with both spiking of mPFC single units and theta-frequency fluctuations in the amplitude of gamma oscillations. Moreover, mPFC theta oscillations were modulated by working memory and synchronized with theta-frequency oscillations in the hippocampus. Finally, both analytical and experimental approaches revealed that this hippocampal-mPFC synchrony relies on activity in the vHPC.

Several lines of evidence have demonstrated that cortical theta can be relevant to behavior. Theta oscillations have been observed previously in area V4 of the primate visual cortex during a visual working memory task (Lee et al., 2005), as well as in the electroencephalogram (EEG) of human subjects performing working memory tasks (Schmiedt et al., 2005; Raghavachari et al., 2006; Haenschel et al., 2009). Of particular interest, theta oscillations at frontal midline sites are a prominent feature of the human EEG (Mitchell et al., 2008) and are modulated by working memory tasks (Womelsdorf et al., 2010). In addition to working memory, theta oscillations in the mPFC have been observed in a number of tasks requiring diverse cognitive functions, such as during instrumental trace conditioning in cats (Paz et al., 2008), during attentional set shifting in rats (Benchenane et al., 2010), and during tests of anxiety in mice (Adhikari et al., 2010; Lesting et al., 2011). mPFC theta oscillations occurring during sleep have also been linked to the consolidation of fear memories (Popa et al., 2010). Although this suggests that theta oscillations are involved in a variety of cognitive and behavioral functions, it is also possible that they reflect common underlying neural computations.

We predicted that, if $\mathrm{mPFC}$ theta oscillations play a role in SWM, their amplitude should be modulated by the mnemonic demands of the SWM task and correlate with the animals' behavior. Our results confirm both of these predictions. Notably, mPFC theta power and phase locking were higher during the choice phase of the task, when animals had to use working memory to guide their decision compared with the sample phase, in which overt behavior was the same but SWM was not required. Conversely, theta power was lower preceding incorrect choices than preceding correct choices. These findings suggest that theta oscillations may facilitate synchrony required for proper task performance. In contrast, we did not find evidence of enhanced synchrony to oscillations in the delta-, beta-, and gammafrequency range, suggesting a frequency-specific modulation of synchrony within the mPFC by SWM. Nevertheless, oscillations at other frequencies in the rodent $\mathrm{mPFC}$ are also likely to play an important role in SWM (Fujisawa and Buzsáki, 2011).

Because the LFP can reflect electrical activity volume conducted from areas distant to the recording site, we sought evidence for theta oscillations in local measures of neural activity. Gamma oscillations are generally thought to reflect synchronization in local microcircuits and are less likely to be volume conducted (Sirota et al., 2008). Therefore, the synchrony we observed between $\mathrm{mPFC}$ theta and gamma oscillations suggests that the theta oscillations also arise locally. The robust modulation of mPFC single-unit activity by local theta oscillations further supports this contention and agrees with MPFC single-unit data in rats (Popa et al., 2010). How do theta oscillations emerge within the $\mathrm{mPFC}$ ? One possibility is that they reflect rhythmic input from the vHPC, which sends excitatory projections to the $\mathrm{mPFC}$ and also displays robust theta oscillations. Our finding that mPFC theta power was not affected by inactivating the vHPC would seem to argue against this possibility, although we cannot exclude that some residual vHPC activity remained that was sufficient to drive theta oscillations in the MPFC. Theta oscillations in the mPFC might also be generated by rhythmic input from structures other than the vHPC. Alternatively, theta oscillations in the mPFC might arise through local mechanisms, for example, through the activity of specific subtypes of interneurons (Beierlein et al., 2000; Blatow et al., 2003). The intrinsic membrane properties of mPFC neurons may also enable them to oscillate preferentially in the theta-frequency range (Dembrow et al., 2010). Interestingly, a computational model of working memory suggests that short-term synaptic plasticity can generate thetafrequency network dynamics under some conditions (Mongillo et al., 2008). Whether these or other mechanisms underlie theta generation in the $\mathrm{mPFC}$ will be important foci for future research.

Given that the hippocampus generates robust theta oscillations (Buzsáki 2002) and is also required for SWM performance, we asked whether hippocampal theta oscillations are modulated by SWM performance. Consistent with previous findings (Jones and Wilson 2005, Montgomery et al., 2009), we found that theta power in the dHPC did not increase during the choice phase. However, we observed a robust increase in vHPC theta power that was comparable with what was observed in the mPFC. Interestingly, theta oscillations in the vHPC, but not the dHPC, also increase during anxiety tasks, in which animals must choose between exploration or safety (Adhikari et al., 2010). The differential modulation observed in these two tasks suggests that theta oscillations in the vHPC are modulated more by behavioral context than theta oscillations in the dHPC, consistent with a body of literature documenting functional differences between the two subregions (Bannerman et al., 2004; Fanselow and Dong, 2010). Furthermore, dHPC theta was qualitatively and quantitatively different from vHPC theta. As we and others have reported (Adhikari et al., 2010; Royer et al., 2010), theta oscillations were of lower magnitude and frequency in the vHPC than in the dHPC. This is unlikely to reflect different recording locations because these differences hold when recordings are restricted to the same hippocampal layers in both dorsal and ventral subdivisions (Adhikari et al., 2010). We also found that mPFC theta was more similar to vHPC than to dHPC theta, consistent with the connectivity between the two regions. However, we cannot exclude the possibility that different recording locations within the $\mathrm{mPFC}$ might have produced different results. Nevertheless, these results 
argue against the possibility that theta oscillations in the mPFC are volume conducted from the hippocampus (Sirota et al., 2008). If that were the case, the frequency of mPFC theta would be expected to be more similar to dHPC theta than vHPC, because the dHPC generates stronger theta oscillations and is closer to the mPFC than the vHPC.

Our study also sought to address how mPFC theta oscillations are synchronized with theta oscillations in the hippocampus. Previous studies reported that, during SWM tasks, neural activity in mPFC and dHPC becomes synchronized in the theta-frequency range, and the degree of synchrony correlates with behavioral performance (Jones and Wilson 2005; Hyman et al., 2010; Sigurdsson et al., 2010). However, because the hippocampal projections to the MPFC originate in the vHPC but not the dHPC (Hoover and Vertes, 2007), it is unclear how theta oscillations in the $\mathrm{dHPC}$ and $\mathrm{mPFC}$ can become synchronized. One possibility is that this is mediated by the vHPC, which is connected to the $\mathrm{dHPC}$ and also projects directly to the mPFC (Dégenètais et al., 2003; Hoover and Vertes, 2007). Our findings are in agreement with such a possibility. First, we observed that dHPC-mPFC and dHPC-vHPC theta coherence was positively correlated. Second, analytically removing the influence of vHPC activity (using partial correlations) dramatically reduced the magnitude of dHPC$\mathrm{mPFC}$ theta power correlations, whereas removing the influence of dHPC did not affect vHPC-mPFC theta power correlations. Finally, pharmacologically inactivating the vHPC decreased theta synchrony between $\mathrm{dHPC}$ and $\mathrm{mPFC}$. These results are consistent with either a permissive role for the vHPC, in which its efferents to the mPFC support hippocampal-prefrontal synchrony, or a direct role in which information flows from the dHPC through the vHPC to the mPFC. Additional experiments will be required to distinguish between these possibilities.

Our findings suggest that, for the dHPC to synchronize with the mPFC, it must synchronize with the vHPC as well. They are consistent with the hypothesis that the vHPC acts as a conduit directly facilitating SWM by relaying spatial information from the dHPC to the mPFC. This hypothesis is supported by studies reporting SWM deficits after inactivation of the $\mathrm{vHPC}$ (Floresco et al., 1997; Wang and Cai, 2006). However, other studies have reported no effect of vHPC inactivation on SWM (Mao and Robinson, 1998; McHugh et al., 2008), perhaps pointing to alternate routes by which the hippocampus interacts with the $\mathrm{mPFC}$. One possibility involves the reuniens nucleus of the thalamus, which has been implicated in tasks requiring the $\mathrm{mPFC}$ and hippocampus (Dolleman-van der Weel et al., 2009; Hembrook et al., 2012; Loureiro et al., 2012). Furthermore, a small percentage of neurons in the reuniens projects to both the $\mathrm{MPFC}$ and hippocampus (Hoover and Vertes, 2012; Varela et al., 2013) that could allow direct facilitation of synchrony between the three structures. Regardless of the contributions other regions may have, our results show that the vHPC plays a notable role in prefrontal-hippocampal coordination. Whether it has an essential function in SWM is an important subject for additional research.

\section{References}

Adhikari A, Topiwala MA, Gordon JA (2010) Synchronized activity between the ventral hippocampus and the medial prefrontal cortex during anxiety. Neuron 65:257-269. CrossRef Medline

Aultman JM, Moghaddam B (2001) Distinct contributions of glutamate and dopamine receptors to temporal aspects of rodent working memory using a clinically relevant task. Psychopharmacology (Berl) 153:353-364. CrossRef Medline

Bannerman DM, Rawlins JN, McHugh SB, Deacon RM, Yee BK, Bast T, Zhang WN, Pothuizen HH, Feldon J (2004) Regional dissociations within the hippocampus—-memory and anxiety. Neurosci Biobehav Rev 28:273-283. CrossRef Medline

Beierlein M, Gibson JR, Connors BW (2000) A network of electrically coupled interneurons drives synchronized inhibition in neocortex. Nat Neurosci 3:904-910. CrossRef Medline

Benchenane K, Peyrache A, Khamassi M, Tierney PL, Gioanni Y, Battaglia FP, Wiener SI (2010) Coherent theta oscillations and reorganization of spike timing in the hippocampal-prefrontal network upon learning. Neuron 66:921-936. CrossRef Medline

Benchenane K, Tiesinga PH, Battaglia FP (2011) Oscillations in the prefrontal cortex: a gateway to memory and attention. Curr Opin Neurobiol 21:475-485. CrossRef Medline

Blatow M, Rozov A, Katona I, Hormuzdi SG, Meyer AH, Whittington MA, Caputi A, Monyer H (2003) A novel network of multipolar bursting interneurons generates theta frequency oscillations in neocortex. Neuron 38:805-817. CrossRef Medline

Buzsáki G (2002) Theta oscillations in the hippocampus. Neuron 33:325340. CrossRef Medline

Colgin LL (2011) Oscillations and hippocampal-prefrontal synchrony. Curr Opin Neurobiol 21:467-474. Medline

Dalley JW, Cardinal RN, Robbins TW (2004) Prefrontal executive and cognitive functions in rodents: neural and neurochemical substrates. Neurosci Biobehav Rev 28:771-784. CrossRef Medline

Dégenètais E, Thierry AM, Glowinski J, Gioanni Y (2003) Synaptic influence of hippocampus on pyramidal cells of the rat prefrontal cortex: an in vivo intracellular recording study. Cereb Cortex 13:782-792. CrossRef Medline

Dembrow NC, Chitwood RA, Johnston D (2010) Projection-specific neuromodulation of medial prefrontal cortex neurons. J Neurosci 30:1692216937. CrossRef Medline

Dolleman-van der Weel MJ, Morris RG, Witter MP (2009) Neurotoxic lesions of the thalamic reuniens or mediodorsal nucleus in rats affect non-mneumonic aspects of watermaze learning. Brain Struct Funct 213: 329-342. CrossRef Medline

Fanselow MS, Dong HW (2010) Are the dorsal and ventral hippocampus functionally distinct structures? Neuron 65:7-19. CrossRef Medline

Floresco SB, Seamans JK, Phillips AG (1997) Selective roles for hippocampal, prefrontal cortical, and ventral striatal circuits in radial-arm maze tasks with or without a delay. J Neurosci 17:1880-1890. Medline

Fries P (2009) Neuronal gamma-band synchronization as a fundamental process in cortical computation. Annu Rev Neurosci 32:209-224. CrossRef Medline

Fujisawa S, Buzsáki G (2011) A $4 \mathrm{~Hz}$ oscillation adaptively synchronizes prefrontal, VTA, and hippocampal activities. Neuron 72:153-165. CrossRef Medline

Gordon JA (2011) Oscillations and hippocampal-prefrontal synchrony. Curr Opin Neurobiol 21:486-491. CrossRef Medline

Haenschel C, Bittner RA, Waltz J, Haertling F, Wibral M, Singer W, Linden DE, Rodriguez E (2009) Cortical oscillatory activity is critical for working memory as revealed by deficits in early-onset schizophrenia. J Neurosci 29:9481-9489. CrossRef Medline

Haupt M, Eccard JA, Winter Y (2010) Does spatial learning ability of common voles (Microtus arvalis) and bank voles (Myodes glareolus) constrain foraging efficiency? Anim Cogn 13:783-791. CrossRef Medline

Hembrook JR, Onos KD, Mair RG (2012) Inactivation of ventral midline thalamus produces selective spatial delayed conditional discrimination impairment in the rat. Hippocampus 22:853-860. CrossRef Medline

Hoover WB, Vertes RP (2007) Anatomical analysis of afferent projections to the medial prefrontal cortex in the rat. Brain Struct Funct 212:149-179. CrossRef Medline

Hoover WB, Vertes RP (2012) Collateral projections from nucleus reuniens of thalamus to hippocampus and medial prefrontal cortex in the rat: a single and doublt retrograde fluorescent labeling study. Brain Struct Funct 217:191-209. CrossRef Medline

Hyman JM, Zilli EA, Paley AM, Hasselmo ME (2010) Working memory performance correlates with prefrontal-hippocampal theta interactions but not with prefrontal neuron firing rates. Front Integr Neurosci 4:2. CrossRef Medline

Izaki Y, Takita M, Akema T (2008) Specific role of the posterior dorsal hippocampus-prefrontal cortex in short-term working memory. Eur J Neurosci 27:3029-3034. CrossRef Medline

Jones MW, Wilson MA (2005) Theta rhythms coordinate hippocampal- 
prefrontal interactions in a spatial memory task. PLoS Biol 3:e402. CrossRef Medline

Jung MW, Qin Y, McNaughton BL, Barnes CA (1998) Firing characteristics of deep layer neurons in prefrontal cortex in rats performing spatial working memory tasks. Cereb Cortex 8:437-450. CrossRef Medline

Lee H, Simpson GV, Logothetis NK, Rainer G (2005) Phase locking of single neuron activity to theta oscillations during working memory in monkey extrastriate visual cortex. Neuron 45:147-156. CrossRef Medline

Leopold DA, Murayama Y, Logothetis NK (2003) Very slow activity fluctuations in monkey visual cortex: implications for functional brain imaging. Cereb Cortex 13:422-433. CrossRef Medline

Lesting J, Narayanan RT, Kluge C, Sangha S, Seidenbecher T, Pape HC (2011) Patterns of coupled theta activity in amygdala-hippocampalprefrontal cortical circuits during fear extinction. PLoS One 6:e21714. CrossRef Medline

Loureiro M, Cholvin T, Lopez J, Merienne N, Latreche A, Cosquer B, Geiger K, Kelche C, Cassel JC, Pereira de Vasconcelos A (2012) The ventral midline thalamus (reuniens and rhomboid nuclei) contributes to the persistence of spatial memory in rats. J Neurosci 32:9947-9959. CrossRef Medline

Mao JB, Robinson JK (1998) Microinjection of GABA-A agonist muscimol into the dorsal but not the ventral hippocampus impairs non-mnemonic measures of delayed non-matching-to-position performance in rats. Brain Res 784:139-147. CrossRef Medline

McHugh SB, Niewoehner B, Rawlins JN, Bannerman DM (2008) Dorsal hippocampal $\mathrm{N}$-methyl-D-aspartate receptors underlie spatial working memory performance during non-matching to place testing on the T-maze. Behav Brain Res 186:41-47. CrossRef Medline

Mitchell DJ, McNaughton N, Flanagan D, Kirk IJ (2008) Frontal-midline theta from the perspective of hippocampal "theta." Prog Neurobiol 86: 156-185. CrossRef

Mongillo G, Barak O, Tsodyks M (2008) Synaptic theory of working memory. Science 319:1543-1546. CrossRef Medline

Montgomery SM, Betancur MI, Buzsáki G (2009) Behavior-dependent coordination of multiple theta dipoles in the hippocampus. J Neurosci 29: 1381-1394. CrossRef Medline
Paz R, Bauer EP, Paré D (2008) Theta synchronizes the activity of medial prefrontal neurons during learning. Learn Mem 15:524-531. CrossRef Medline

Popa D, Duvarci S, Popescu AT, Léna C, Paré D (2010) Coherent amgdalocortical theta promotes fear memory consolidation during paradoxical sleep. Proc Natl Acad Sci U S A 107:6516-6519. CrossRef Medline

Raghavachari S, Lisman JE, Tully M, Madsen JR, Bromfield EB, Kahana MJ (2006) Theta oscillations in human cortex during a working-memory task: evidence for local generators. J Neurophysiol 95:1630-1638. CrossRef Medline

Royer S, Sirota A, Patel J, Buzsáki G (2010) Distinct representations and theta dynamics in dorsal and ventral hippocampus. J Neurosci 30: 1777-1787. CrossRef Medline

Schmiedt C, Brand A, Hildebrandt H, Basar-Eroglu C (2005) Event-related theta oscillations during working memory tasks in patients with schizophrenia and healthy controls. Brain Res Cogn Brain Res 25:936-947. CrossRef Medline

Siapas AG, Lubenov EV, Wilson MA (2005) Prefrontal phase locking to hippocampal theta oscillations. Neuron 46:141-151. CrossRef Medline

Sigurdsson T, Stark KL, Karayiorgou M, Gogos JA, Gordon JA (2010) Impaired hippocampal-prefrontal synchrony in a genetic mouse model of schizophrenia. Nature 464:763-767. CrossRef Medline

Sirota A, Montgomery S, Fujisawa S, Isomura Y, Zugaro M, Buzsáki G (2008) Entrainment of neocortical neurons and gamma oscillations by the hippocampal theta rhythm. Neuron 60:683-697. CrossRef Medline

Varela C, Kumar S, Yang JY, Wilson MA (2013) Anatomical substrates for direct interactions between hippocampus, medial prefrontal cortex, and the thalamic nucleus reuniens. Brain Struct Funct. Advance online publication. Retrieved July 31, 2013. doi:10.1007/s00429-013-0543-5. CrossRef Medline

Wang GW, Cai JX (2006) Disconnection of the hippocampal-prefrontal cortical circuits impairs spatial working memory performance in rats. Behav Brain Res 175:329-336. CrossRef Medline

Womelsdorf T, Vinck M, Leung LS, Everling S (2010) Selective thetasynchronization of choice-relevant information subserves goal-directed behavior. Front Hum Neurosci 4:210. CrossRef Medline 